\title{
Simplified DM models with the full SM gauge symmetry: the case of $t$-channel colored scalar mediators
}

\author{
P. Ko, ${ }^{a, b}$ Alexander Natale, ${ }^{a}$ Myeonghun Park $^{c}$ and Hiroshi Yokoya ${ }^{b}$ \\ ${ }^{a}$ School of Physics, KIAS, \\ Seoul 02455, Korea \\ ${ }^{b}$ Quantum Universe Center, KIAS, \\ Seoul 02455, Korea \\ ${ }^{c}$ Center for Theoretical Physics of the Universe, IBS, \\ Daejeon 34051, Korea \\ E-mail: pko@kias.re.kr, alexnatale@kias.re.kr, hyokoya@kias.re.kr, \\ parc.ctpu@gmail.com
}

ABSTRACT: The general strategy for dark matter (DM) searches at colliders currently relies on simplified models. In this paper, we propose a new $t$-channel UV-complete simplified model that improves the existing simplified DM models in two important respects: (i) we impose the full SM gauge symmetry including the fact that the left-handed and the righthanded fermions have two independent mediators with two independent couplings, and (ii) we include the renormalization group evolution when we derive the effective Lagrangian for DM-nucleon scattering from the underlying UV complete models by integrating out the $t$-channel mediators. The first improvement will introduce a few more new parameters compared with the existing simplified DM models. In this study we look at the effect this broader set of free parameters has on direct detection and the mono- $X+$ MET $(X=$ jet, $W, Z$ ) signatures at $13 \mathrm{TeV}$ LHC while maintaining gauge invariance of the simplified model under the full SM gauge group. We find that the direct detection constraints require DM masses less than $10 \mathrm{GeV}$ in order to produce phenomenologically interesting collider signatures. Additionally, for a fixed mono- $\mathrm{W}$ cross section it is possible to see very large differences in the mono-jet cross section when the usual simplified model assumptions are loosened and isospin violation between RH and LH DM-SM quark couplings are allowed.

Keywords: Beyond Standard Model, Gauge Symmetry

ARXIV EPRINT: 1605.07058 


\section{Contents}

1 Introduction 1

$2 t$-channel UV completion with colored scalar mediators 5

$\begin{array}{lll}3 & \text { Direct detection } & 7\end{array}$

4 Collider signatures $\quad 11$

4.1 Mono- $W+$ missing $E_{T} \quad 12$

$\begin{array}{lll}4.2 & \text { Mono-jet }+ \text { missing } E_{T} & 14\end{array}$

5 Collider results \& discussion $\quad 15$

$\begin{array}{lll}6 & \text { Summary } & 22\end{array}$

\section{Introduction}

The astrophysical evidence for the existence of Dark Matter (DM) is convincing, but the properties of DM remain largely unknown [1]. In an effort to elucidate the properties of DM there are several predominant strategies: indirect detection experiments which search for DM annihilation signals, direct detection via nuclear recoil experiments such as LUX, and search strategies at colliders where DM is directly produced and observed via large missing transverse momentum $\left(E_{T}\right)$. When investigating direct detection signals the effective field theory (EFT) approach is a sensible way to describe the interaction of DM with the detector while utilizing only two free parameters; the scale of the new physics that mediates this DM-SM interaction $\left(\Lambda_{i}\right.$ which is much larger than the hadronic energy scale) and the DM mass $\left(m_{\chi}\right)$. The lowest dimensional effective Lagrangian for DM direct detection (DD) can be written schematically as

$$
\mathcal{L}_{\mathrm{DD}}=\sum_{i} \frac{1}{\Lambda_{i}^{2}} \bar{q} \Gamma_{i} q \bar{\chi} \Gamma_{i} \chi(+H . c .) .
$$

Colliders searches for mono $X+\mathbb{E}_{T}$ signatures (with $X=W, g, \gamma, Z$ ) at the LHC have also used this EFT approach during Run-I [2-6], but have also utilized UV-complete models such as Supersymmetry [7].

However, at the center-of-mass energies at the LHC this EFT approach would break down $[8,9]$, which warrants the use of UV-complete models at the expense of introducing many more free parameters. An alternative approach is the utilization of so-called simplified DM models [10]. These simplified DM models generically satisfy a few criteria [11, 12]: the simplified models should involve a particle stable enough so it may produces a large 
$E_{T}$ signature, the simplified model should respect the unbroken SM gauge group at minimum and it should not violate approximate and global symmetries of the SM, with the ultimate goal of describing interesting collider phenomenology involving $\mathbb{E}_{T}$ while keeping the number of free parameters to a minimum. Then the above effective Lagrangian for DM DD is modified as

$$
\frac{1}{\Lambda_{i}^{2}} \bar{q} \Gamma_{i} q \bar{\chi} \Gamma_{i} \chi \rightarrow \frac{g_{q} g_{\chi}}{m_{\phi}^{2}-s} \bar{q} \Gamma_{i} q \bar{\chi} \Gamma_{i} \chi
$$

when we consider the $s$-channel UV completion for $q \bar{q} \rightarrow \phi \rightarrow \chi \bar{\chi}$.

However this strategy with simplified DM models have ample room for improvement in two important respects. First of all, the simplified models do not respect the full SM gauge invariance, which may be problematic when they are adopted to DM search studies at high energy colliders. At the LHC CM energy, one has to respect the full SM gauge symmetry, and not just the unbroken subgroup of it. Recently, importance of the full SM invariance, unitarity and gauge invariance with respect to the mediators was noticed in a few independent studies [13-15], which will be detailed in the subsequent discussions. When we impose the full SM gauge symmetry, we have to realize that the SM fermions have two independent chiralities, left-handed $(\mathrm{LH})$ and right-handed $(\mathrm{RH})$, and SM gauge interactions are chiral as well. Therefore the LH and the RH fermions $\left(f_{L}\right.$ and $\left.f_{R}\right)$ would have independent mediators, $\widetilde{f}_{L}$ and $\widetilde{f}_{R}$, with independent masses $\left(m_{\tilde{f}_{L}}\right.$ and $\left.m_{\tilde{f}_{R}}\right)$ and two independent Yukawa couplings $\left(\lambda_{L}\right.$ and $\left.\lambda_{R}\right)$ to the SM fermions.

Let us illustrate the main point with the $\bar{q} q \bar{\chi} \chi$ opeartor [14]. The $s$-channel UV completion of this operator involves an additional singlet scalar $S$ and its mixing with the SM Higgs field. The above operator would be generated from two gauge invariant and renormalizable operators, $\overline{Q_{L}} H d_{R}$ and $S \bar{\chi} \chi$ followed by the $h-s$ mixing after $H$ and $S$ develop nonzero VEVs. ${ }^{1}$ Then the amplitude for $q \bar{q} \rightarrow \chi \bar{\chi}$ UV completion involves two scalar bosons in the intermediate states:

$$
\frac{1}{s-m_{1}^{2}+i m_{1} \Gamma_{1}}-\frac{1}{s-m_{2}^{2}+i m_{2} \Gamma_{2}}
$$

where $m_{1}=125 \mathrm{GeV}$ is the observed $125 \mathrm{GeV}$ SM Higgs-like scalar boson. Two scalar propagators with a definite relative sign and magnitude leads to different collider signatures both at the LHC $\left(p p \rightarrow\right.$ monojet + missing $E_{T}, t \bar{t}+$ missing $\left.E_{T}\right)[14,17]$ and at the ILC $\left(e^{+} e^{-} \rightarrow Z^{0}+\right.$ missing $\left.E_{T}\right)$ [18]. Note that the usual $s$-channel UV completion in terms of a single propagator does not hold for the (scalar $) \times($ scalar $)$ operator, in sharp constrast with naive expectation for a single propagator in eq. (1.2).

The same is true for the $t$-channel mediator case too. In this case, the LH quark and the RH quark would couple to two different colored mediators, $\widetilde{q}_{L}$ and $\widetilde{q}_{R}$ with two independent couplings $\lambda_{L}$ and $\lambda_{R}$ (see section 2 for the $t$-channel UV complete Lagrangian and more precise definitions of these parameters, and also Feynman diagrams in figures 4, 5 and 6 in section 4 ). Then the UV completion generically calls for two independent propagators of $\widetilde{q}_{L}$

\footnotetext{
${ }^{1}$ Note that $S \bar{q} q$ or $h \bar{\chi} \chi$ violate the SM gauge symmetry and renormalizability, and we have to consider UV completions of these operators. Introducing an additional singlet scalar $S$ as in refs. [14, 16] is a minimal UV completion where DM $\chi$ is a pure singlet fermion.
} 
and $\widetilde{q}_{R}$, instead of a single propagator, eq. (1.2). Only the case of $W+\mathbb{E}_{T}$ would involve a single propagator, because $W$ couples only to the LH quark and its partner mediator. This phenomena is due to the facts that (i) the SM fermions in 4-dim spacetime have two independent chiralities, (ii) the SM gauge theory is chiral, and (iii) the full SM gauge symmetry is imposed on the UV completions. Then the simplified DM models proposed in this paper would not violate gauge invariance and unitarity. Otherwise one could get physically nonsensible results.

Secondly, there is a technical issue when one derives the effective Lagrangian suitable for direct detection of DM. One can integrate out the mediator at the mediator mass scale, obtaining 4-fermion operators. However the relevant energy scale for the DM direct detection cross section is order of nuclear energy scale, and one has to include the renormalization effects from the mediator mass scale down to the nuclear energy scale. ${ }^{2}$ This procedure was not included properly in the simplified DM models [21], and should be performed before one derives the constraints on the simplified DM models from the DM direct detection data. This can be included in a straightforward manner using the renormalization-group analysis for the DM-nucleon scattering. ${ }^{3} \mathrm{RG}$ evolution can not only change the effective coupling strengths at different energy scale, but also generate new operators that were not present when the mediators were integrated out at the mediator mass scales [23]. Due to this second effect, there would be more than one effective operator at nucleon mass scale that are relevant to DM DD. In other words, It is not realistic at all to assume that DM DD can be described by a single effective operator, in sharp contrast to what earlier literatures did assume using the effective Lagrangian for the DM DD.

In this paper, we propose a minimal simplified DM model that respects the full SM gauge symmetry, assuming the DM is a Dirac fermion $\chi$ with negative dark $Z_{2}$ parity and introducing $t$-channel colored mediators. Imposing the full SM gauge symmetry in the DMSM interaction Lagrangian is the new and the unique aspect of our proposal, and improves the earlier attempts for simplified DM models for collider searches and direct detection of DM. The number of new parameters in the simplified DM models with the full SM gauge symmetry is usually "four", one more than the simplified models in the literature [24]. One extra parameter is coming from the second mass scale, which often enters in the Lagrangian when we impose the full SM gauge symmetry. This feature has not been noticed in earlier literature, and the interpretation of DM search at colliders and in direct detection experiments is modified when this is taken into account. In addition, we include the RG running effects when we derive the effective Lagrangian for DM-nucleon scattering and compare with the bounds from LUX and other DM direct detection experiments.

In the construction of simplified DM models with the full SM gauge symmetry, we impose the following conditions to the model Lagrangian:

- Invariance under the full SM gauge symmetry: in many DM models, one often imposes the invariance of the model only under the unbroken SM gauge symmetry,

\footnotetext{
${ }^{2}$ This is well known from flavor physics ( $K, B$ physics, see ref. [19] for example) as well as top forwardbackward asymmetry [20].

${ }^{3}$ Recently, this issue has been pointed out in ref. [22] in the context of the DM simplified models with $s$-channel vector mediators.
} 
$H_{\mathrm{SM}} \equiv \mathrm{SU}(3)_{C} \times \mathrm{U}(1)_{\mathrm{em}}$. This may be acceptable for studying direct detection of $\mathrm{DM}$, but not for collider signatures of DM. However, at high energy colliders, one has to impose the full SM gauge symmetry, $G_{\mathrm{SM}} \equiv \mathrm{SU}(3)_{C} \times \mathrm{SU}(2)_{L} \times \mathrm{U}(1)_{Y}$. The importance of taking into account $\mathrm{SU}(2)_{L}$ gauge invariance when investigating $\mathrm{DM}$ signatures at colliders has previously been pointed out in a recent paper [15], where a potential enhancement to the mono- $W$ signature was previously found when considering unequal mediator couplings to up and down quarks [25], but this enhancement was found to result from spurious longitudinal $\mathrm{W}$ boson contributions [15]. The important point is that the EFT method can break down at a scale on order of the VEV, well before the scale $\Lambda$ as generally assumed [15].

- Issue of dark (gauge) symmetry responsible for absolute stability or longevity of DM particle: from the previous discussion on the $G_{\mathrm{SM}}$ vs. $H_{\mathrm{SM}}$, it is also clear that the model Lagrangian and phenomenology thereof would depend strongly on what dark (gauge) symmetry we assume is responsible for the DM stability or longevity. Since we don't know anything about the dark sector at the moment, we will make the simplest working assumption that DM in our model carries $Z_{2}$-odd parity, whereas all the SM particles are even under $Z_{2}$.

It is natural to assume that DM may have some kind of (its own) gauge symmetries [26-38]. And then there could be other extra dark fields (such as dark Higgs or dark gauge fields) which might be not that heavy and so we may have to include them in our simplified models. This part will be highly model dependent, and we make a simple assumption they are all heavy enough so that we can ignore them in our simplified model.

- Renormalizability and unitarity: in EFT approaches to DM, it is common to consider higher dimensional nonrenormalizable operators. This approach is a fine starting point, especially for the DM direct detection. However one has to think about the UV completions eventually, and there could be more than one UV completion that leads to the same low energy EFT at a given order. Many DM simplified models start from DM direct detection and then extrapolate to collider signatures at higher energies. However, in a series of papers on Higgs portal DM models, it has been shown that the EFT can give us completely misleading results compared with the full renormalizable and unitary DM models, in the context of singlet fermion DM $[16,39]$ and vector DM [27, 40, 41] with Higgs portal interactions. Since we can easily miss important phenomenology within EFT which is nonrenormalizable and nonunitary (see for example refs. [18, 42]), we will start from renormalizable and unitary DM models. ${ }^{4}$

- Totalitarian principle: we will allow all possible interactions that are consistent with the assumed symmetries. This is important for real phenomenology since we cannot

\footnotetext{
${ }^{4}$ In this paper, we consider the colored scalar mediators in the $t$-channel. If we consider the vector mediators in the $s$-channel, we have to address the issue of gauge anomaly cancellation, which was discussed in ref. [13].
} 
control the chiralities of the initial partons at hadron colliders. Note that $W+E_{T}$ will involve the LH fermions and their dark mediators, whereas $g+\mathbb{E}_{T}$ and $\gamma+\mathbb{E}_{T}$ will involve both the LH and the RH fermions in the initial states with equal strengths. And $Z+\mathbb{E}_{T}$ also involves both chiralities but with different strengths. Therefore it is not realistic to analyze $W+\mathbb{E}_{T}, g+\mathbb{E}_{T}, \gamma+\mathbb{E}_{T}$ and $Z+\mathbb{E}_{T}$ within simplified models with a single $t$-channel mediator. This condition is independent of unitarity or gauge symmetry, and will allow us to analyze collider in more general contexts.

- Flavor physics: if the mediator carries nontrivial SM gauge charges (such as color and/or electric charges), the one loop diagrams involving the DM and the mediators may generate the nontrivial FCNC, which would be strongly constrained by various data from the $K, B$ meson systems. For the case of Dirac fermion DM, the constraints are weaker than the case of the Majorana fermion DM, since there is no chirality flip in the loop.

Based on these assumptions, we construct minimal simplified DM models with full SM gauge symmetry. In this paper, we shall concentrate on the $t$-channel colored mediators in $q \bar{q} \rightarrow \chi \bar{\chi}$.

This paper is organized as follows. In section 2, we show the simplified DM models with the full SM gauge invariance as well as renormalizability and unitarity. In section 3, we derive the effective Lagrangian relevant for DM direct detection by integrating out the colored scalar mediators and performing the RG evolution down to the nucleon mass scale, and discuss that there appear a number of different operators appears simultanesouly. In particular isospin violation would be generic because of two independent scalar mediators originating from two different chirality of the SM fermions. In section 4, we derive the amplitudes for mono $X+E_{T}$ with $X=W, g$, and present the numerical analysis and the releted phenomenogy in section 5 . Then we summarize in section 6 .

\section{$2 t$-channel UV completion with colored scalar mediators}

Let us consider the $t$-channel UV completion with scalars. We introduce 3 types of new scalar bosons, $\widetilde{Q}_{L i}, \widetilde{u}_{R i}$ and $\widetilde{d}_{R i}$ with negative $Z_{2}$ parity, which are partners of $Q_{L i} \equiv$ $\left(u_{L i}, d_{L i}\right)^{T}, u_{R i}$ and $d_{R i}$ respectively. ${ }^{5}$ Simplified models with colored scalar mediators that couple to the quarks have been previously studied [24, 25, 43-47], however these studies have assumed either just an up-like SU(2) singlet [45], a down-like singlet [44], a doublet $[46,48]$, or a simplified model similar to our proposal however with universal couplings to all generations and universal masses for up-like and down-like scalars [24, 49, 50]. The gauge invariant interaction Lagrangian between quarks and DM in our model is given by:

$$
\mathcal{L}_{\mathrm{t}-\text { channel }}=-\left[\bar{\chi} \widetilde{Q}_{L}^{i \dagger}\left(\lambda_{Q_{L}}\right)_{i}{ }^{j} Q_{L j}+\bar{\chi} \widetilde{u}_{R}^{i \dagger}\left(\lambda_{u_{R}}\right)_{i}{ }^{j} u_{R j}+\bar{\chi} \widetilde{d}_{R}^{i \dagger}\left(\lambda_{d_{R}}\right)_{i}{ }^{j} d_{R j}+H . c .\right]
$$

\footnotetext{
${ }^{5}$ In this paper, we consider only the scalar partners of the SM quarks. It would be straightforward to introduce the scalar partners of the SM leptons.
} 
We also show the Lagrangian for the newly introduced scalar fields:

$$
\begin{aligned}
\mathcal{L}_{\text {scalar }}= & D_{\mu} \widetilde{Q}_{L}^{i^{\dagger}} D^{\mu} \widetilde{Q}_{L i}-\widetilde{Q}_{L}^{i \dagger}\left[\left(m_{\widetilde{Q}_{L}, 0}^{2}\right)_{i}^{j}+2\left(\lambda_{Q_{L} H}\right)_{i}^{j} H^{\dagger} H\right] \widetilde{Q}_{L j} \\
& +D_{\mu} \widetilde{u}_{R}^{i \dagger} D^{\mu} \widetilde{u}_{R i}-\widetilde{u}_{R}^{i \dagger}\left[\left(m_{\widetilde{u}_{R}, 0}^{2}\right)_{i}^{j}+2\left(\lambda_{u_{R} H}\right)_{i}{ }^{j} H^{\dagger} H\right] \widetilde{u}_{R j} \\
& +D_{\mu} \widetilde{d}_{R}^{i \dagger} D^{\mu} \widetilde{d}_{R i}-\widetilde{d}_{R}^{i \dagger}\left[\left(m_{\widetilde{d}_{R}, 0}^{2}\right)_{i}^{j}+2\left(\lambda_{d_{R} H}\right)_{i}{ }^{j} H^{\dagger} H\right] \widetilde{d}_{R j} \\
& -\left[\widetilde{Q}_{L}^{i \dagger}\left(A_{u}\right)_{i}{ }^{j} \widetilde{H}^{j} \widetilde{u}_{R j}+\widetilde{Q}_{L}^{i \dagger}\left(A_{d}\right)_{i}{ }^{j} H \widetilde{d}_{R j}+H . c\right] \\
& -\lambda_{\widetilde{q}_{L}}\left(\widetilde{Q}_{L}^{\dagger} \widetilde{Q}_{L}\right)^{2}-2 \lambda_{4} H^{\dagger} \widetilde{Q}_{L} \widetilde{Q}_{L}^{\dagger} H
\end{aligned}
$$

where the covariant derivative contains all the SM gauge fields according to the SM gauge quantum numbers of the fields upon which $D_{\mu}$ acts. At this level, all the fields are in the interaction eigenstates.

The matrices $m_{\widetilde{Q}_{L}}^{2}, \lambda_{Q_{L} H}, m_{\widetilde{u}_{R}}^{2}, \lambda_{u_{R} H}, m_{\widetilde{d}_{R}}^{2}$ and $\lambda_{d_{R} H}$ are Hermitian matrices in flavor space. We have suppressed the scalar partners of the SM leptons, for which there could be similar terms.

Scalar quark masses are given by

$$
\begin{aligned}
& m_{\widetilde{u}_{L}}^{2}=m_{\widetilde{Q}_{L}, 0}^{2}+\lambda_{Q_{L} H} v^{2} \\
& m_{\widetilde{d}_{L}}^{2}=m_{\widetilde{Q}_{L}, 0}^{2}+\lambda_{Q_{L} H} v^{2}+\lambda_{4} v^{2}=m_{\widetilde{u}_{L}}^{2}+\lambda_{4} v^{2} \\
& m_{\widetilde{u}_{R}}^{2}=m_{\widetilde{u}_{R}, 0}^{2}+\lambda_{u_{R} H} v^{2} \\
& m_{\widetilde{d}_{R}}^{2}=m_{\widetilde{d}_{R}, 0}^{2}+\lambda_{d_{R} H} v^{2}
\end{aligned}
$$

Note that the $\lambda_{4}$ term induces the mass splitting between $\widetilde{u}_{L}$ and $\widetilde{d}_{L}$ :

$$
m_{\widetilde{d}_{L}}^{2}-m_{\widetilde{u}_{L}}^{2}=\lambda_{4} v^{2}
$$

thereby generating isospin violation effects at colliders and at DM direct detections. The trilinear $A_{u, d}$ terms generate the left-right mass mixing between $\widetilde{u}_{L}$ and $\widetilde{u}_{R}$ (and also between $\widetilde{d}_{L}$ and $\widetilde{d}_{R}$ ). Throughout this paper we use the notation familiar from an EFT description of our model where $\Lambda_{Q_{L}}^{-1}=\lambda_{Q_{L}} / m_{\widetilde{Q}_{L}}, \Lambda_{u_{R}}^{-1}=\lambda_{u_{R}} / m_{\widetilde{u}_{R}}$, and $\Lambda_{d_{R}}^{-1}=\lambda_{d_{R}} / m_{\widetilde{d}_{R}}$.

After EWSB, we have to rotate both quarks and their scalar partners to the mass eigenstates. The resulting Lagrangian will be similar to the above one, except that

$$
H^{\dagger} H \rightarrow \frac{v^{2}}{2}\left(1+\frac{h}{v}\right)^{2}
$$

Note that the $m^{2}$ and $\lambda$ would not be simultaneously diagonalizable in general. Therefore one would have flavor violation in the Higgs couplings to the scalar partners of $Q_{L}, u_{R}$ and $d_{R}$, which would lead to rare Higgs decays into

$$
H \rightarrow \widetilde{q}_{i}^{*} \widetilde{q}_{j}^{*} \rightarrow\left(\bar{q}_{i}+\chi\right)+\left(q_{j}+\bar{\chi}\right)
$$


Also the scalar partners of the SM quarks will modify $H \rightarrow g g, \gamma \gamma, Z \gamma$ through loop effects. The deviations of the Higgs signal strengths from the SM values will depend on the ratio of the Higgs contribution to the mass of the scalar partners of the SM fermions.

Basically this model for $\lambda_{4}=0$ is similar to the MSSM in the decoupling limit of the Higgs sector and heavy gaugino/Higgsino limit, except that there is only one neutral Dirac fermion in the dark sector (analogous to the lightest neutralino in the MSSM) and not four neutral Majorana fermions in our model. The colored scalar mediators are the similar to the scalar quarks in MSSM. Therefore the usual scalar quark search bounds from monojet $+\mathbb{E}_{T}$ and dijet $+\mathbb{E}_{T}$ could be reinterpreted in the framework of our model, after including the correction factors for Dirac vs. Majorana DM.

Once we include 3 generations of dark scalar partners, their mass matrices would not be diagonal in the basis where quark masses are diagonal. This misalignment of mass matrices in the flavor space would lead to flavor and CP violation induced by dark scalars, similarly to the gluino-mediated FCNC and CP violation in the general MSSM. One crucial difference exists in this model, since the DM is a Dirac fermion and not a Majorana fermion there is no chirality flip inside the loops, and the usual FCNC constraints become weaker in our model compared to the MSSM.

\section{Direct detection}

Let us derive the effective Lagrangian describing the direct detection cross section for the DM-nucleon scattering. Note that there are a number of different effective operators generated simultaneously if we integrate out the dark scalars, $\widetilde{Q}_{L}, \widetilde{u}_{R}$ and $\widetilde{d}_{R}$. The Wilson coefficient of the effective operators depend on a number of parameters, including three different mass scales of dark scalars, and there is no single mass scale we can associate with a single effective operator, in sharp contrast to the conventional wisdom. This is due to the condition that the DM interactions with the SM fermions respects the full SM gauge symmetry. Both collider searches and direct detection of $\chi$ depend on at least two different and independent mass scales.

The resulting effective Lagrangian for dark matter direct detection is given by

$$
\begin{aligned}
\mathcal{L}_{\mathrm{DD}}= & -\frac{\left|\left(\lambda_{q_{L}}\right)_{1}{ }^{1}\right|^{2}}{m_{\tilde{u}_{L}}^{2}} \bar{\chi} u_{L} \bar{u}_{L} \chi-\frac{\left|\left(\lambda_{q_{L}}\right)_{1}{ }^{1}\right|^{2}}{m_{\tilde{d}_{L}}^{2}} \bar{\chi} d_{L} \bar{d}_{L} \chi-\frac{\left|\left(\lambda_{u_{R}}\right)_{1}{ }^{1}\right|^{2}}{m_{\tilde{u}_{R}}^{2}} \bar{\chi} u_{R} \bar{u}_{R} \chi \\
& -\frac{\left|\left(\lambda_{d_{R}}\right)_{1}{ }^{1}\right|^{2}}{m_{\tilde{d}_{R}}^{2}} \bar{\chi} d_{R} \bar{d}_{R} \chi-\frac{\left|\left(\lambda_{q_{L}}\right)_{2}{ }^{2}\right|^{2}}{m_{\tilde{s}_{L}}^{2}} \bar{\chi} s_{L} \bar{s}_{L} \chi-\frac{\left|\left(\lambda_{d_{R}}\right)_{2}{ }^{2}\right|^{2}}{m_{\tilde{s}_{R}}^{2}} \bar{\chi} s_{R} \bar{s}_{R} \chi
\end{aligned}
$$

After Fierz transformation, the above Lagrangian is cast into the following form:

$$
\begin{aligned}
\mathcal{L}_{\mathrm{DD}}= & \frac{\left|\left(\lambda_{q_{L}}\right)_{1}{ }^{1}\right|^{2}}{2 m_{\tilde{u}_{L}}^{2}} \bar{\chi}_{R} \gamma_{\mu} \chi_{R} \bar{u}_{L} \gamma^{\mu} u_{L}+\frac{\left|\left(\lambda_{q_{L}}\right)_{1}{ }^{1}\right|^{2}}{2 m_{\tilde{d}_{L}}^{2}} \bar{\chi}_{R} \gamma_{\mu} \chi_{R} \bar{d}_{L} \gamma^{\mu} d_{L} \\
& +\frac{\left|\left(\lambda_{u_{R}}\right)_{1}{ }^{1}\right|^{2}}{2 m_{\tilde{u}_{R}}^{2}} \bar{\chi}_{L} \gamma_{\mu} \chi_{L} \bar{u}_{R} \gamma^{\mu} u_{R}+\frac{\left|\left(\lambda_{d_{R}}\right)_{1}{ }^{1}\right|^{2}}{2 m_{\tilde{d}_{R}}^{2}} \bar{\chi}_{L} \gamma_{\mu} \chi_{L} \bar{d}_{R} \gamma^{\mu} d_{R} \\
& +\frac{\left|\left(\lambda_{q_{L}}\right)_{2}{ }^{2}\right|^{2}}{2 m_{\tilde{s}_{L}}^{2}} \bar{\chi}_{R} \gamma_{\mu} \chi_{R} \bar{s}_{L} \gamma^{\mu} s_{L}+\frac{\left|\left(\lambda_{d_{R}}\right)_{2}{ }^{2}\right|^{2}}{2 m_{\tilde{s}_{R}}^{2}} \bar{\chi}_{L} \gamma_{\mu} \chi_{L} \bar{s}_{R} \gamma^{\mu} s_{R}
\end{aligned}
$$


Since the strange quark current does not contribute to the nucleon matrix element, we can ignore the strange quark and concentrate only on the 1st generation quarks. Therefore we shall suppress the generation indices on the matrices $\lambda$ 's, and make abbreviations: $\lambda_{q}, \lambda_{u}$ and $\lambda_{d}$ from now on for the DM direct detection.

We have worked in the leading order in QCD, ignoring the $\chi \chi G_{\mu}^{a} G^{a \mu \nu}$ and twist-2 operators that could contribute to the DM direct detection. It would be straightforward to include them in the analysis of DM direct detection, which have been calculated explicitly for Majorana DM [51-53], and can be used without modification for Dirac DM [54].

Because new physics generates a number of effective operators simultaneously in general, it is unrealistic to assume that, for example, new physics generates only $\left(\bar{\chi} \gamma_{\mu} \chi\right)\left(\bar{q} \gamma^{\mu} q\right)$ or $(\bar{\chi} \chi)(\bar{q} q)$. Considering the effective operators suitable for describing DM direct detection as starting points, one can miss important pieces of new physics at colliders regarding the DM sector.

We have assumed that the couplings $\lambda_{q_{L}}, \lambda_{u_{R}}$ and $\lambda_{d_{R}}$ are flavor diagonal in the mass eigenstates in order to avoid the bounds from the FCNC. We also assume that the $\widetilde{d}$ and $\widetilde{s}$ are degenerate in order to avoid the constraints from $K^{0}-\overline{K^{0}}$ mixing. These assumptions are rather ad hoc, but do not violate the underlying gauge symmetry, and thus are theoretically consistent assumptions. They could be somewhat relaxed within the current constraints from the FCNC in the $K$ and $B$ meson systems compared with the SUSY case, since we assume that the DM is a Dirac fermion, and not a Majorana fermion, unlike the $N=1$ SUSY models.

Then the direct detection cross sections from DM-N scattering (with $N=p, n$ ) are given by

$$
\begin{aligned}
\sigma_{N}^{\mathrm{SI}}= & \frac{1}{64 \pi} \frac{m_{N}^{2} m_{\chi}^{2}}{\left(m_{\chi}+m_{N}\right)^{2}}\left[\left(\frac{3\left|\lambda_{Q_{L}}\right|^{2}}{2 m_{\tilde{Q}_{L}}^{2}}+\frac{\left|\lambda_{u_{R}}\right|^{2}}{2 m_{\tilde{u}_{R}}^{2}}+\frac{2\left|\lambda_{d_{R}}\right|^{2}}{2 m_{\tilde{d}_{R}}^{2}}\right)\right. \\
& \left.+\frac{Z}{A}\left(\frac{\left|\lambda_{u_{R}}\right|^{2}}{2 m_{\tilde{u}_{R}}^{2}}-\frac{\left|\lambda_{d_{R}}\right|^{2}}{2 m_{\tilde{d}_{R}}^{2}}\right)\right]^{2},
\end{aligned}
$$

under the assumption that $\lambda_{4}=0$. As discussed numerous places in the literature [23, $52,55-57]$, there are potentially important direct detection effects due to RGE running from the UV to the nuclear scale. A user-friendly procedure for approximating these effects is presented in ref. [23], and this process generically induces extra dependencies on the mediator mass in the SI cross section and can induce isospin-violating effects and different structures of operators than what occurs at the scale of the mediator mass. By implementing the procedure outlined in ref. [23] to account for the running of the EFT from the scale $\Lambda$ to the hadronic scale, and taking into account the latest results from LUX [58, 59], it is found that for dark matter masses in the range $10 \mathrm{GeV}<m_{\chi}<$ $1000 \mathrm{GeV}$, our simplified model is essentially excluded for any choice of $\lambda_{q_{i}}$ and $m_{\widetilde{q}_{i}}$ that would be phenomenologically interesting at colliders. Note that the relic density for GeV scale DM is generically too large for TeV mass scalars [43], and too low when the DM is $\mathrm{TeV}$ scale, however the tension with the relic density can potentially be alleviated with couplings to the leptons and still evade the LUX constraints [54]. However, the direct 
detection and relic density constraints were found assuming $\chi$ is absolutely stable, and is the only DM particle. In scenarios where $\chi$ is only stable long enough to escape detection at a collider, or where there are multiple particles responsible for the total DM energy density in the universe, then the constraints from direct detection and the relic density of DM can be relaxed. Specifically, in the case where there are two DM species, the direct detection cross section will depend on the relative density of each DM species divided by its mass times the relevant cross section [60]. Under such assumptions, it is possible that the $\chi$ which produces the interesting collider signatures is an order of $100 \mathrm{~s}$ of $\mathrm{GeV}$ while avoiding direct detection and relic density constraints, and in this case the earlier assumption that the mediators which allow coupling to heavier quarks are much larger than the other mediators, allows for the Fermi-LAT constraints on $b \bar{b}$ annhilation to be satisfied [61]. Note that in the case where $m_{\chi}<10 \mathrm{GeV}$, the indirect detection constraints are important and would need to be considered in the case where interactions are added, however for the purposes of the paper we do not specify any interactions beyond the $t$ channel mediators. Additional contributions to the direct detection cross section can also include contributions from gluons and the electric/magnetic dipole moments, however the gluon contribution to direct detection found in ref. [52] is proportional to $\alpha_{s} \frac{m_{\chi}}{m_{\tilde{q}_{i}}^{4}}$, thus in the low DM mass region $\left(m_{\chi}<10 \mathrm{GeV}\right)$ the gluon operator is suppressed, and the dipole moment contributions to direct detection are small when there is a tree-level coupling to up and down quarks [54] as is the case in our model. There is also a contribution to the gluon operator from the heavy quarks, however our model has dirac fermions and our assumptions about flavor structure means these heavy quark contributions to the gluon operators depend on the 'heavy' quark flavored mediators, which can be safely taken to be much more massive than the other mediators, so these contributions proportional to $m_{t}$ can be suppressed.

Generic isospin violation is also present (even when $\lambda_{4}=0$ ) due to the unbalanced $\lambda_{u_{R}}$ and $\lambda_{d_{R}}$, which is consistent with the underlying SM gauge symmetry. Due to the generic isospin violation, and the mixing of different operators due to running effects noted earlier, it is not legitimate to consider only one mediator mass, and in general we have to allow all three different mediators for direct detection (and generally non-zero values for $\lambda_{4}$ ). That is, even if a particular set of EFT operators at the EW or TeV scale is assumed, the running effects generically produce a mixture of the EFT operators [23, 55]. As discussed in refs. [23, 55], these running effects are important for two main reasons: (1) they are found to have a quantitatively significant effect on the resulting cross section, (2) not only can they introduce new operators that are missing from the UV (for instance generating spin independent operators in a model that only has a UV spin dependent operator), but depending on the EW structure of the mediators these running effects will produce Wilson coefficients at the nucleon energy that are a non-trivial mixture of the mediators. In figure 1 the direction detection cross section, with full RGE running effects taken into account through the procedure outlined in ref. [23], is shown versus the $m_{\chi}$. The dark scalar couplings are assumed to be $\lambda_{Q_{L}}=\lambda_{u_{R}}=1$ and $\lambda_{d_{R}}=\lambda_{4}=0$. CDMS Lite Run 1 and Lux direct detection constraints are plotted, along with the coherent neutrino 


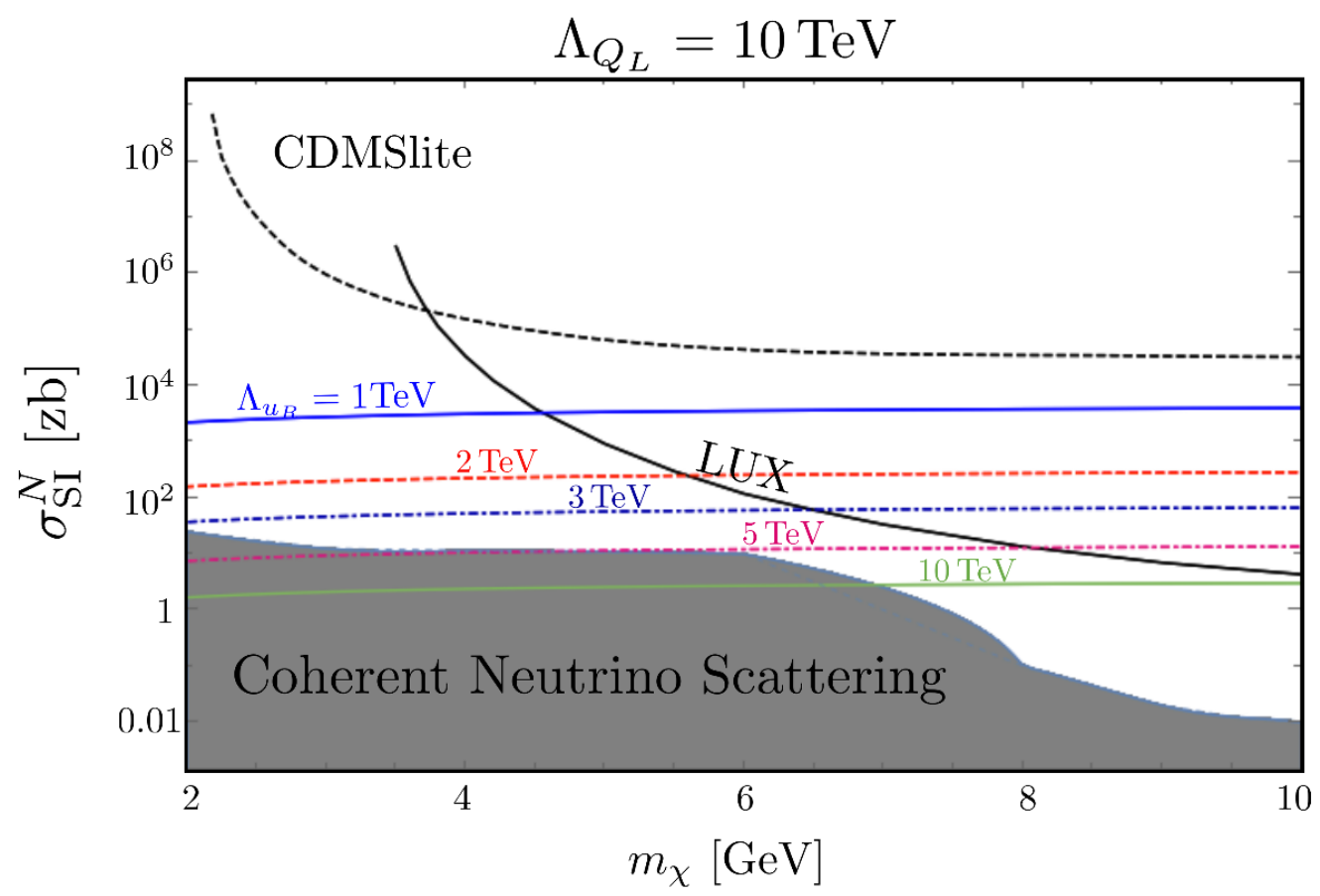

Figure 1. Low DM mass spin-independent WIMP-Nucleon scattering constraints for $\lambda_{Q_{L}}=\lambda_{u_{R}}=$ 1 and $\lambda_{d_{R}}=0$. Effects from running are accounted for using the procedure outlined in ref. [23].

scattering background, which severely constrain $m_{\chi}$ if we assume $\chi$ is absolutely stable and the major source of the DM energy density. Let us define effective new physics scales for this model:

$$
\Lambda_{Q_{L}} \equiv \frac{m_{\widetilde{Q}_{L}}}{\lambda_{Q_{L}}}, \quad \Lambda_{u_{R}} \equiv \frac{m_{\widetilde{u}_{R}}}{\lambda_{u_{R}}}, \quad \Lambda_{d_{R}} \equiv \frac{m_{\widetilde{d}_{R}}}{\lambda_{d_{R}}} .
$$

These three scales can independently take different values without violating the SM gauge symmetry. Then it is important to note that for a fixed $\Lambda_{Q_{L}}$ variations in $\lambda_{u_{R}}$ change the direct detection cross-section by almost four orders of magnitude in this low $m_{\chi}$ region. Even in the simplifying assumption where the doublet is mostly decoupled (i.e., $\Lambda_{Q_{L}}>$ $10 \mathrm{TeV}$ ) and $\Lambda_{u_{R}}=\Lambda_{d_{R}}=1 \mathrm{TeV}$, the SI cross section is close to the limit from Run 1 CDMSlite [62]. Importantly, the generic isospin violating effect leads to a potentially large material dependence, for instance using eq. (3.3), and the $\mathrm{Z}$ and $\mathrm{A}$ values for Xenon and Germanium, the relative difference in the cross sections $\left(\frac{\sigma_{S I}^{X e}-\sigma_{S I}^{G e}}{\sigma_{S I}^{G e}}\right)$ is given by:

$$
\Delta \sigma / \sigma=\frac{-76 \Lambda_{Q_{L}}^{2}\left(\Lambda_{d_{R}}^{2}-\Lambda_{u_{R}}^{2}\right)\left(1684 \Lambda_{d_{R}}^{2} \Lambda_{Q_{L}}^{2}+7074 \Lambda_{d_{R}}^{2}+1853 \Lambda_{Q_{L}}^{2} \Lambda_{u_{R}}^{2}\right)}{17161\left(13 \Lambda_{d_{R}}^{2} \Lambda_{Q_{L}}^{2}+54 \Lambda_{d_{R}}^{2} \Lambda_{u_{R}}^{2}+14 \Lambda_{Q_{L}}^{2} \Lambda_{u_{R}}^{2}\right)^{2}}
$$

where $\Lambda_{q_{i}}=m_{\widetilde{q}_{i}} / \lambda_{q_{i}}$. This equation becomes zero if $\Lambda_{u_{R}}=\Lambda_{d_{R}}$, or if both singlet EFT scales are taken to infinity (i.e., the singlets are entirely decoupled). However, in the limit where $\Lambda_{Q_{L}}, \Lambda_{u_{R}} \rightarrow \infty$, eq. (3.4) yields a positive value of $0.0419\left(\sigma_{S I}^{X e}>\sigma_{S I}^{G e}\right)$, where as $\Lambda_{Q_{L}}, \Lambda_{d_{R}} \rightarrow \infty$ produces a value on order of $-0.0441\left(\sigma_{S I}^{X e}<\sigma_{S I}^{G e}\right)$. The exact 

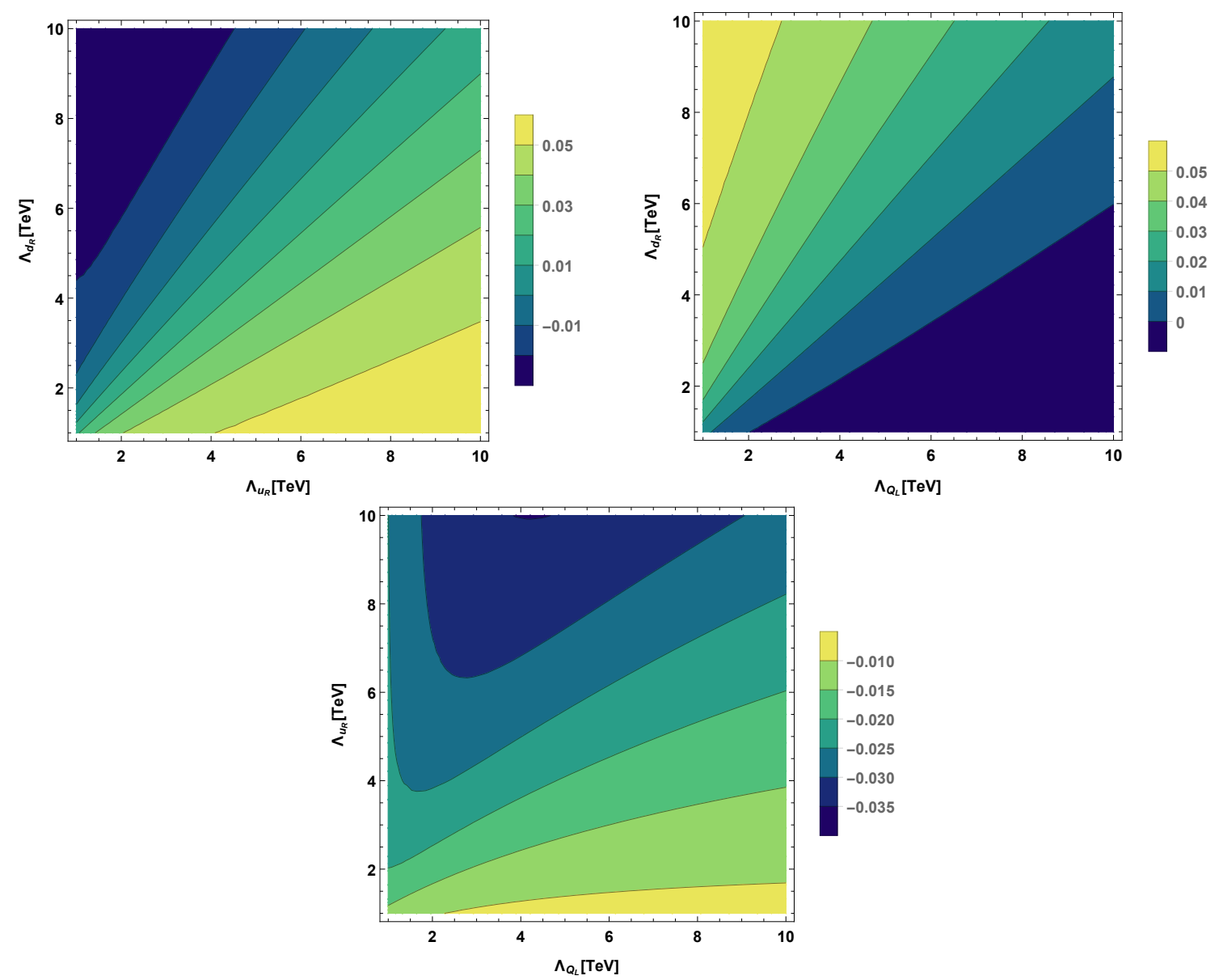

Figure 2. $\Delta \sigma / \sigma$ with running effects with one mediator scale entirely decoupled, $\Lambda_{d_{R}}^{-1}=\lambda_{d_{R}} / m_{\widetilde{d}_{R}}$, and $\lambda_{4}$.

expression for the isospin violating effects is modified from running effects, which can be significant [23]. In figure 2 a contour plot of $\Delta \sigma / \sigma$ is shown in the limit where $\widetilde{u}_{R}, \widetilde{d}_{R}$ and $\widetilde{Q}_{L}$ are decoupled respectively, and the coupling constants $\left(\lambda_{i}\right)$ are assumed to be equal to unity. The maximum positive value is found to be greater than 0.05 for $\Delta \sigma / \sigma$, which is larger than the limiting case, but this is due to the running effects which induce a small isospin violating effect on otherwise non-isospin violating simplified models [23]. Thus, if it was found that there was no relative difference between Xe and Ge DM cross sections, this could potentially indicate some underlying isospin violation and the existence of coupling to left-handed ( $\mathrm{LH})$ and right-handed $(\mathrm{RH})$ quarks, as the slight miss-match in scalar masses is actually required to cancel the isospin violating effects found in [23]. Figure 3 shows the case for finite fixed values of $\Lambda_{Q_{L}}$, and $\lambda_{i}$ 's are again set to unity for simplicity. Note the generally non-linear relationship between $\Lambda_{u_{R}}$ and $\Lambda_{d_{R}}$ that is required to entirely eliminate isospin violating effects in direct detection.

\section{Collider signatures}

The direct detection experiments probe the mediators in combination of $\lambda^{2} / m_{\text {mediator }}^{2}$, with some additional $\lambda$ dependence from the running effects [23], whereas colliders can probe 

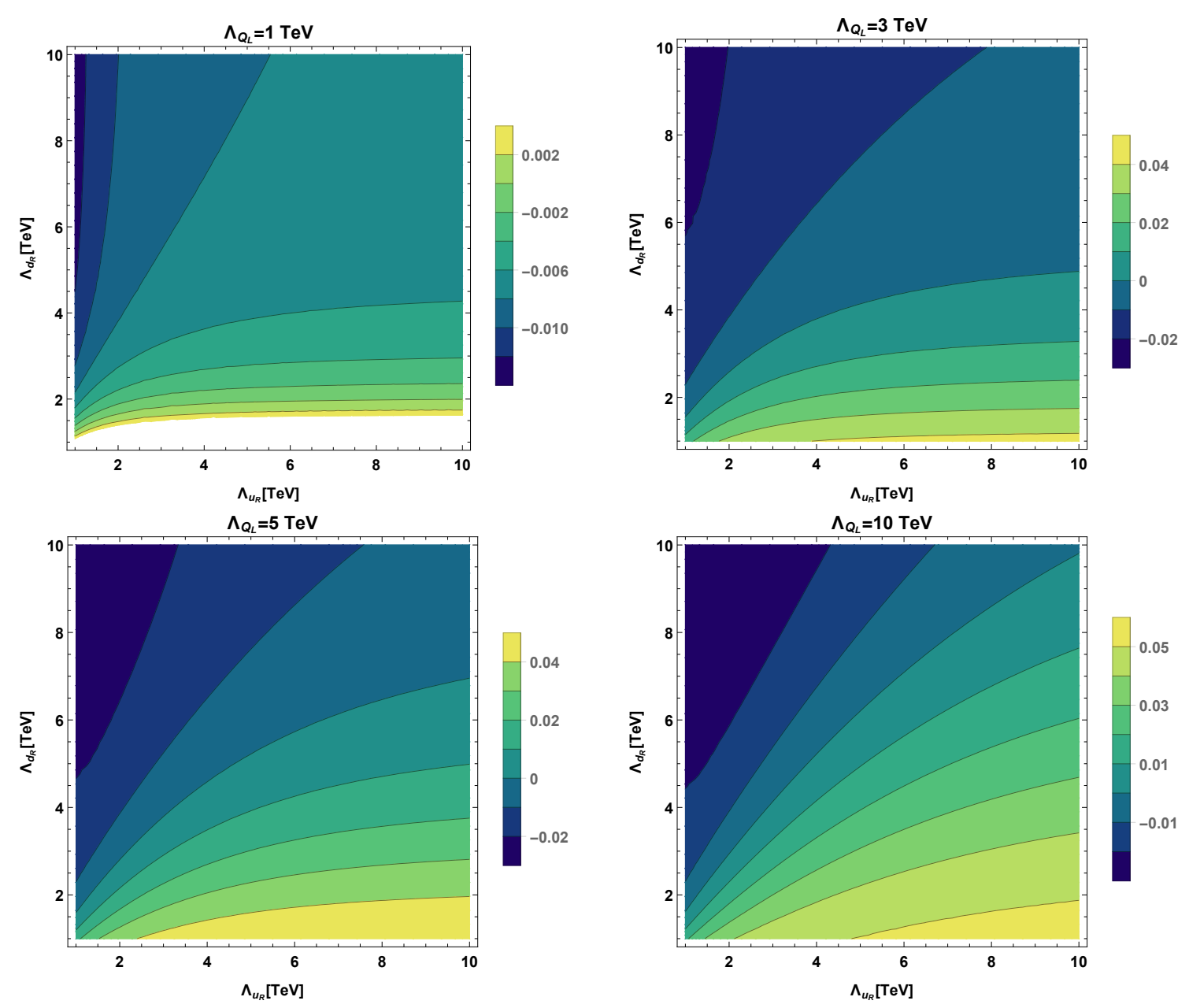

Figure 3. $\Delta \sigma / \sigma$ with running effects for fixed $\Lambda_{Q_{L}}$ and $\lambda_{4}=0$.

$m_{\text {mediator }}$ directly if the mediators can be pair-produced and decay into dijet $+\mathscr{E}_{T}$. While the flavor structure in this model is more general than the MSSM, the simplified SUSY searches for squark pair-production and subsequent decay to jets and missing energy will provide direct constraints to our model. By choosing the minimum mediator mass to be above the limits set from the simplified model searches at the LHC, these constraints can be taken into account and the differences between our model and existing simplified models can be emphasized. If the scalars cannot be pair-produced, then the mono- $\mathrm{X}+\mathbb{E}_{T}$ signatures ( $\mathrm{X}=\mathrm{g}, \mathrm{W}, \mathrm{Z}$, etc.) will constrain $\lambda^{2} / m_{\text {mediator }}^{2}$ as direct detection experiments do. In the following, we will see that mono- $W+\mathbb{E}_{T}$ is unique in that it probes only one mass scale $\widetilde{Q}_{L}$, because of the LH interactions of $W$ with the SM fermions. Other cases always involve both the LH and the RH fermions and their dark scalar partners, and thus depend on at least two independent mass scales. Therefore it is important to study mono- $W+\mathbb{E}_{T}$, since it can separately probe the LH sector only.

\subsection{Mono- $W+$ missing $E_{T}$}

As stated earlier, the mono- $W$ mode is nice in that it probes the LH quark sector only, since only $\widetilde{Q}_{L 1} \equiv\left(\widetilde{u}_{L}, \widetilde{d}_{L}\right), \widetilde{Q}_{L 2} \equiv\left(\widetilde{c}_{L}, \widetilde{s}_{L}\right)$ and $\widetilde{Q}_{L 3} \equiv\left(\widetilde{t}_{L}, \widetilde{b}_{L}\right)$ contribute (the top contri- 



Figure 4. Feynman diagrams for mono- $W$ that produce $\mathcal{O}(6)$ EFT operators.

bution would be negligible for the $13 \mathrm{TeV}$ LHC). At parton level, there are three Feynman diagrams that contribute to the processes $u \bar{d} \rightarrow W^{+}+\chi \bar{\chi}$, however as pointed out in the literature the internal Bremsstrahlung occurs at a higher order in an EFT [15], and large isospin violating effects between $\widetilde{Q}_{L u}$ and $\widetilde{Q}_{L d}$ due to $\lambda_{4} \neq 0$ provide only a very small enhancement of the mono- $W$ relative to mono-jet [63], so for the purposes of this paper only the processes depicted in figure 4 were analyzed after verifying the internal Bremsstrahlung and isospin violation, due to the $\lambda_{4}$ mass-splitting, were small.

In our simplified model, the parton level amplitude is given by

$$
\mathcal{M}=\left(\mathcal{M}_{a}^{\mu}+\mathcal{M}_{b}^{\mu}+\mathcal{M}_{c}^{\mu}\right) \epsilon_{\mu}^{*}(q),
$$

where

$$
\begin{aligned}
\mathcal{M}_{a}^{\mu} & =\frac{g_{w} \lambda_{Q_{L}}^{2}}{\sqrt{2}} \frac{\bar{v}\left(p_{2}\right) \gamma^{\mu} P_{L}\left(\not q-\not p_{2}\right) v\left(k_{2}\right) \bar{u}\left(k_{1}\right) P_{L} u\left(p_{1}\right)}{\left(p_{2}-q\right)^{2}\left(\left(p_{1}-k_{1}\right)^{2}-m_{\widetilde{Q}_{L}}^{2}\right)}, \\
\mathcal{M}_{b}^{\mu} & =\frac{g_{w} \lambda_{Q_{L}}^{2}}{\sqrt{2}} \frac{\bar{v}\left(p_{2}\right) P_{R} v\left(k_{2}\right) \bar{u}\left(k_{1}\right) P_{L}\left(\not p_{1}-\not q\right) \gamma^{\mu} u\left(p_{1}\right)}{\left(p_{1}-q\right)^{2}\left(\left(p_{2}-k_{2}\right)^{2}-m_{\widetilde{Q}_{L}}^{2}\right)},
\end{aligned}
$$

and

$$
\mathcal{M}_{c}^{\mu}=\frac{g_{w} \lambda_{Q_{L}}^{2}}{\sqrt{2}} \frac{\bar{v}\left(p_{2}\right) P_{R} v\left(k_{2}\right) \bar{u}\left(k_{1}\right) P_{L} u\left(p_{1}\right)}{\left(\left(p_{1}-k_{1}\right)^{2}-m_{\widetilde{Q}_{L}}^{2}\right)\left(\left(p_{2}-k_{2}\right)^{2}-m_{\widetilde{Q}_{L}}^{2}\right)}\left(2 k_{1}-2 p_{1}-q\right)^{\mu}
$$

After suitable Fierz transformation $[64,65]$ these amplitudes can be written as:

$$
\mathcal{M}_{a}^{\mu}=\frac{g_{w} \lambda_{Q_{L}}^{2}}{2 \sqrt{2}} \frac{\bar{v}\left(p_{2}\right) \gamma^{\mu} P_{L}\left(\not 1-\not p_{2}\right) \gamma_{\alpha} u\left(p_{1}\right) \bar{u}\left(k_{1}\right) P_{L} \gamma^{\alpha} v\left(k_{2}\right)}{\left(p_{2}-q\right)^{2}\left(\left(p_{1}-k_{1}\right)^{2}-m_{\widetilde{Q}_{L}}^{2}\right)}
$$



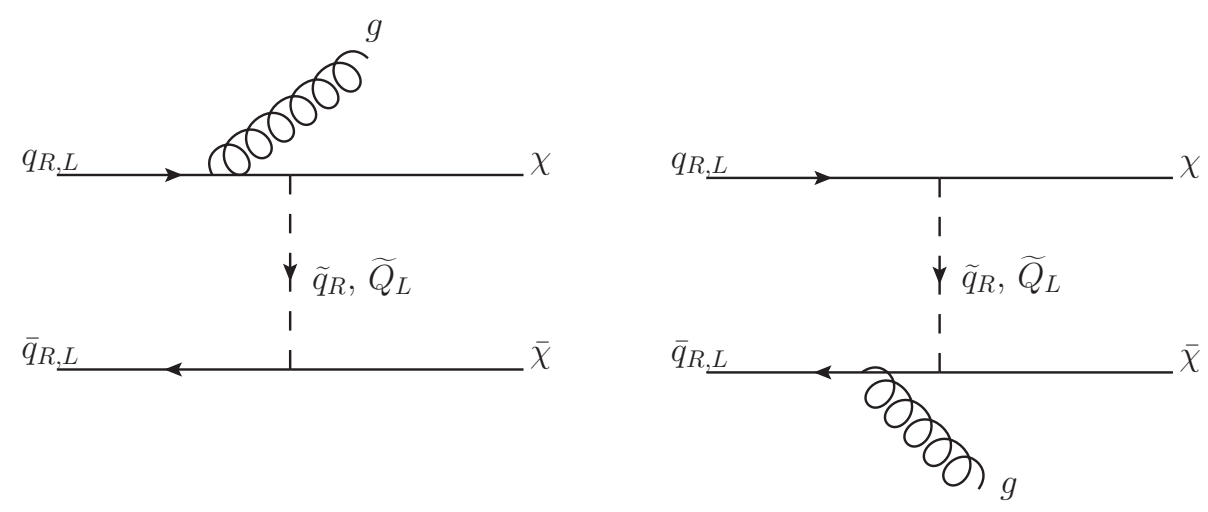

Figure 5. Feynman diagrams for mono gluon that produce $\mathcal{O}(6)$ EFT operators.

$$
\mathcal{M}_{b}^{\mu}=\frac{g_{w} \lambda_{Q_{L}}^{2}}{2 \sqrt{2}} \frac{\bar{v}\left(p_{2}\right) \gamma_{\alpha} P_{L}\left(\not p_{1}-\not q\right) \gamma^{\mu} u\left(p_{1}\right) \bar{u}\left(k_{1}\right) P_{L} \gamma^{\alpha} v\left(k_{2}\right)}{\left(p_{1}-q\right)^{2}\left(\left(p_{2}-k_{2}\right)^{2}-m_{\widetilde{Q}_{L}}^{2}\right)}
$$

and

$$
\mathcal{M}_{c}^{\mu}=\frac{g_{w} \lambda_{Q_{L}}^{2}}{2 \sqrt{2}} \frac{\bar{v}\left(p_{2}\right) \gamma_{\alpha} P_{L} u\left(p_{1}\right) \bar{u}\left(k_{1}\right) P_{L} \gamma^{\alpha} v\left(k_{2}\right)}{\left(\left(p_{1}-k_{1}\right)^{2}-m_{\widetilde{Q}_{L}}^{2}\right)\left(\left(p_{2}-k_{2}\right)^{2}-m_{\widetilde{Q}_{L}}^{2}\right)}\left(2 k_{1}-2 p_{1}-q\right)^{\mu}
$$

In the limit $m_{\widetilde{Q}_{L}} \rightarrow \infty$, the above amplitude is simplified as

$$
\mathcal{M}_{\mathrm{EFT}}=\mathcal{M}_{\mathrm{EFT}}^{\mu} \epsilon_{\mu}^{*}(q),
$$

where

$$
\begin{aligned}
\mathcal{M}_{\mathrm{EFT}}^{\mu}= & \frac{-g_{w} \lambda_{Q_{L}}^{2}}{2 \sqrt{2} m_{\widetilde{Q}_{L}}^{2}}\left(\frac{\bar{v}\left(p_{2}\right) \gamma^{\mu} P_{L}\left(\not p-\not p_{2}\right) \gamma_{\alpha} u\left(p_{1}\right) \bar{u}\left(k_{1}\right) P_{L} \gamma^{\alpha} v\left(k_{2}\right)}{\left(p_{2}-q\right)^{2}}\right. \\
& \left.+\frac{\bar{v}\left(p_{2}\right) \gamma_{\alpha} P_{L}\left(\not p_{1}-\not q\right) \gamma^{\mu} u\left(p_{1}\right) \bar{u}\left(k_{1}\right) P_{L} \gamma^{\alpha} v\left(k_{2}\right)}{\left(p_{1}-q\right)^{2}}\right)
\end{aligned}
$$

which can be derived from the effective Lagrangian from eq. (3.2) only the LH quark terms. Hence, this process depends only one mass scale $m_{\widetilde{Q}_{L}}$ and one Yukawa coupling $\lambda_{\widetilde{Q}_{L}}$.

\subsection{Mono-jet + missing $E_{T}$}

Since it is very difficult determining a quark-jet from a gluon-jet, the mono-jet channel includes both mono- $g+\mathbb{E}_{T}$ and mono- $q+\mathbb{E}_{T}$ channels. The Feynman diagrams that contribute to the lowest order in the EFT theory for each separate channel are illustrated in figure 5 and figure 6 .

In this channel both the $\mathrm{LH}$ and the $\mathrm{RH}$ quarks contribute with equal weights.

The parton level amplitude for

$$
q \bar{q} \rightarrow g+\chi \bar{\chi}
$$


(with $q=u, d, s, c, b$ ) is given by

$$
\begin{aligned}
\mathcal{M} & =\left(\mathcal{M}_{a, L}^{\mu}+\mathcal{M}_{b, L}^{\mu}+\mathcal{M}_{c, L}^{\mu}+\mathcal{M}_{a, R}^{\mu}+\mathcal{M}_{b, r}^{\mu}+\mathcal{M}_{c, R}^{\mu}\right) \epsilon_{\mu}^{*}(q), \\
\mathcal{M}_{a, L}^{\mu} & =\frac{g_{s} \lambda_{Q_{L}}^{2}}{2} \frac{\bar{v}\left(p_{2}\right) \gamma^{\mu} P_{L} \phi_{a} \gamma_{\alpha} u\left(p_{1}\right) \bar{u}\left(k_{1}\right) P_{L} \gamma^{\alpha} v\left(k_{2}\right)}{\left(p_{2}-q\right)^{2}\left(\left(p_{1}-k_{1}\right)^{2}-m_{\widetilde{Q}_{L}}^{2}\right)} \\
\mathcal{M}_{b, L}^{\mu} & =\frac{g_{s} \lambda_{Q_{L}}^{2}}{2} \frac{\bar{v}\left(p_{2}\right) \gamma_{\alpha} P_{L} \phi_{b} \gamma^{\mu} u\left(p_{1}\right) \bar{u}\left(k_{1}\right) P_{L} \gamma^{\alpha} v\left(k_{2}\right)}{\left(p_{1}-q\right)^{2}\left(\left(p_{2}-k_{2}\right)^{2}-m_{\widetilde{Q}_{L}}^{2}\right)} \\
\mathcal{M}_{c, L}^{\mu} & =\frac{g_{s} \lambda_{Q_{L}}^{2}}{2} \frac{\bar{v}\left(p_{2}\right) \gamma_{\alpha} P_{L} u\left(p_{1}\right) \bar{u}\left(k_{1}\right) P_{L} \gamma^{\alpha} v\left(k_{2}\right)}{\left(\left(p_{1}-k_{1}\right)^{2}-m_{\widetilde{Q}_{L}}^{2}\right)\left(\left(p_{2}-k_{2}\right)^{2}-m_{\widetilde{Q}_{L}}^{2}\right)}\left(2 k_{1}-2 p_{1}-q\right)^{\mu} \\
\mathcal{M}_{a, R}^{\mu} & =\frac{g_{s} \lambda_{q_{R}}^{2}}{2} \frac{\bar{v}\left(p_{2}\right) \gamma^{\mu} P_{R} \phi_{a} \gamma_{\alpha} u\left(p_{1}\right) \bar{u}\left(k_{1}\right) P_{R} \gamma^{\alpha} v\left(k_{2}\right)}{\left(p_{2}-q\right)^{2}\left(\left(p_{1}-k_{1}\right)^{2}-m_{\widetilde{q}_{R}}^{2}\right)} \\
\mathcal{M}_{b, R}^{\mu} & =\frac{g_{s} \lambda_{q_{R}}^{2}}{2} \frac{\bar{v}\left(p_{2}\right) \gamma_{\alpha} P_{R} \phi_{b} \gamma^{\mu} u\left(p_{1}\right) \bar{u}\left(k_{1}\right) P_{R} \gamma^{\alpha} v\left(k_{2}\right)}{\left(p_{1}-q\right)^{2}\left(\left(p_{2}-k_{2}\right)^{2}-m_{\widetilde{q}_{R}}^{2}\right)} \\
\mathcal{M}_{c, R}^{\mu} & =\frac{g_{s} \lambda_{q_{R}}^{2}}{2} \frac{\bar{v}\left(p_{2}\right) \gamma_{\alpha} P_{R} u\left(p_{1}\right) \bar{u}\left(k_{1}\right) P_{R} \gamma^{\alpha} v\left(k_{2}\right)}{\left(\left(p_{1}-k_{1}\right)^{2}-m_{\widetilde{q}_{R}}^{2}\right)\left(\left(p_{2}-k_{2}\right)^{2}-m_{\widetilde{q}_{R}}^{2}\right)}\left(2 k_{1}-2 p_{1}-q\right)^{\mu}
\end{aligned}
$$

In the limit $m_{\widetilde{Q}_{L}} \rightarrow \infty$, the above amplitude is simplifed as

$$
\begin{aligned}
\mathcal{M}_{\mathrm{EFT}}= & \frac{-g_{s}}{2}\left[\frac { \lambda _ { Q _ { L } } ^ { 2 } } { m _ { \widetilde { Q } _ { L } } ^ { 2 } } \left(\frac{\bar{v}\left(p_{2}\right) \gamma^{\mu} P_{L} \phi_{a} \gamma_{\alpha} u\left(p_{1}\right) \bar{u}\left(k_{1}\right) P_{L} \gamma^{\alpha} v\left(k_{2}\right)}{\left(p_{2}-q\right)^{2}}\right.\right. \\
& \left.+\frac{\bar{v}\left(p_{2}\right) \gamma_{\alpha} P_{L} \phi_{b} \gamma^{\mu} u\left(p_{1}\right) \bar{u}\left(k_{1}\right) P_{L} \gamma^{\alpha} v\left(k_{2}\right)}{\left(p_{1}-q\right)^{2}}\right) \\
& +\frac{\lambda_{q_{R}}^{2}}{m_{\widetilde{q}_{R}}^{2}}\left(\frac{\bar{v}\left(p_{2}\right) \gamma^{\mu} P_{R} \phi_{a} \gamma_{\alpha} u\left(p_{1}\right) \bar{u}\left(k_{1}\right) P_{R} \gamma^{\alpha} v\left(k_{2}\right)}{\left(p_{2}-q\right)^{2}}\right. \\
& \left.\left.+\frac{\bar{v}\left(p_{2}\right) \gamma_{\alpha} P_{R} \phi_{b} \gamma^{\mu} u\left(p_{1}\right) \bar{u}\left(k_{1}\right) P_{L} \gamma^{\alpha} v\left(k_{2}\right)}{\left(p_{1}-q\right)^{2}}\right)\right]
\end{aligned}
$$

which can be derived from the effective Lagrangian in eq. (3.2).

The mono-quark channel Feynman diagrams are shown in figure 6, where higher order EFT terms are neglected. In this channel, the width of the mediator potentially becomes important, and has been included when calculating the full mono-jet cross section. While higher multiplicity jet events are included in LHC jet searches, these were not calculated for this paper. Because both the mono-gluon and mono-quark channels depend on all three mediators, and their couplings, certain assumptions must be made in order to make LHC predictions.

\section{Collider results \& discussion}

In this section, we present collider phenomenology of mono- $X+\operatorname{missing} E_{T}$ based on the simplified models with SM gauge symmetry, where $X=W / Z$, or jet. Contributions to 

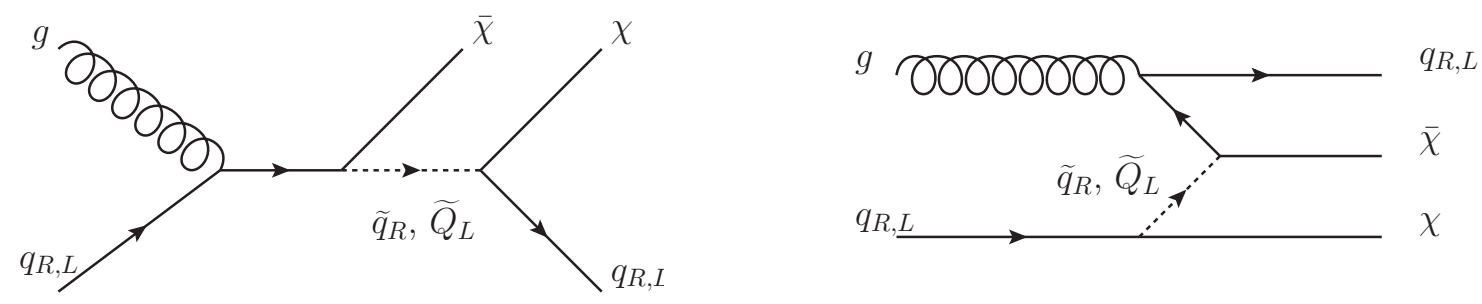

Figure 6. Feynman diagrams for $\mathrm{g} \mathrm{q} \rightarrow \mathrm{q} \chi \chi$ process at $\mathcal{O}(6)$ in an EFT.

the mono- $Z+\mathbb{E}_{T}$ signature includes both the LH and RH quarks, which contribute with different weights as determined by the $\mathrm{SU}(2)_{L} \times \mathrm{U}(1)_{Y}$ charges of $u_{L, R}$ and $d_{L, R}$, thus it does not probe a single scale and has been included in the collider signature analysis as it may provide complementary information to the mono- $W$ and mono-jet signatures. In order to perform the collider signature analysis our model is implemented in Feynrules [66], where the hadronic level cross section is calculated in Madgraph 5 [67] utilizing the NNPDF23 parton distribution function set [68]. Kinematic plots are then produced through the Pythia and Delphes interfaces [67, 69, 70] and analyzed in Root [71]. For the mono-jet search, a minimum jet $p_{T}$ of $100 \mathrm{GeV}$ is used with a pseudo-rapidity cut of $|\eta|<5$, where both the mono-g and mono-quark channels are included. The minimum scalar mass is taken to be $1 \mathrm{TeV}$ to account for the dijet $+\mathscr{E}_{T}$ searches at the $13 \mathrm{TeV}$ LHC [72]. The hadronic level cross section for the mono- $W^{+}$channel is plotted in figure 7 for varying $m_{Q_{L}}$ and fixed values of $\Lambda_{Q_{L}}$ with $m_{\chi}=5 \mathrm{GeV}$, and the cross section for the mono-jet channel (including both gluon and quark jets) is plotted in figure 8 with the assumption that $\lambda_{d_{R}}=0, \Lambda_{u_{R}}=10 \mathrm{TeV}, m_{\chi}=5 \mathrm{GeV}$, and $\lambda_{4}=0$ for varying $m_{Q_{L}}$ and fixed values of $\Lambda_{Q_{L}}$. We find that the mono- $W$ cross sections are almost flat, and thus are well described by the EFT with the cut-off parameter $\Lambda$ except when $m_{Q_{L}}$ is close to the dijet limit, where the cross section is lower than the EFT prediction. This is because the correspondence of $\Lambda_{L} \leftrightarrow m_{\widetilde{Q}_{L}} / f_{L}$ is violated in the scattering amplitudes due to the typical virtuality of an order of a few $\mathrm{TeV}$ of the $t$-channel mediator. We find a similar tendency in the low mediator mass regions in the mono- $Z$ cross sections, which are shown in figure 9 with the assumptions made for mono-jet channel in figure 8. On the other hand, for the mono-jet channel the cross section is enhanced in small $m_{\widetilde{Q}_{L}}$ due to the $s$-channel pole. By setting $\lambda_{4}=0$ the parameter space we scan over would be equivalent to MSSM models where the $\tilde{u}_{L}$ and $\tilde{d}_{L}$ are degenerate, $\tilde{u}_{R}$ and $\tilde{d}_{R}$ are allowed to independently vary, while the chargino spectrum is such that the squarks decay almost entirely to a light quark and the LSP, but where the third-generation squarks are largely decoupled.

Note that the mono- $W$ signature is generically small at the $13 \mathrm{TeV}$ LHC, and so while it provides a weaker constraint on $\Lambda$ compared to the mono-jet, it does uniquely provide a constraint on $\Lambda_{Q_{L}}$. The contour plot in figure 10 shows the mono- $W$, mono- $Z$ and monojet cross sections infb for $m_{\widetilde{Q}_{L}}$ versus $m_{\widetilde{u}_{R}}$ where $\lambda_{Q_{L}}=\lambda_{u_{R}}=1, \lambda_{d_{R}}=\lambda_{4}=0$, and $m_{\chi}=5 \mathrm{GeV}$. 




Figure 7. Hadron level cross section for $\mathrm{pp} \rightarrow W+\mathbb{E}_{T}$ at $13 \mathrm{TeV}$.



Figure 8. Hadron level cross section for $\mathrm{pp} \rightarrow J+\mathbb{E}_{T}$ at $13 \mathrm{TeV}$. 


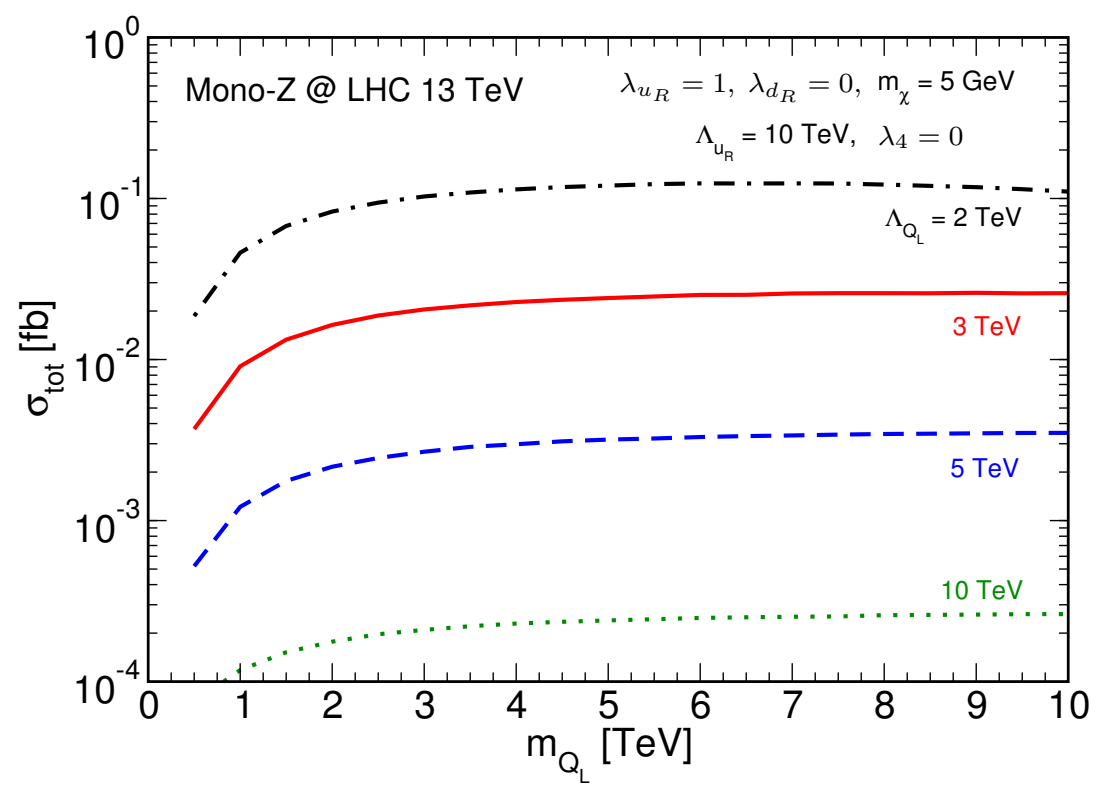

Figure 9. Hadron level cross section for $\mathrm{pp} \rightarrow Z+E_{T}$ at $13 \mathrm{TeV}$.

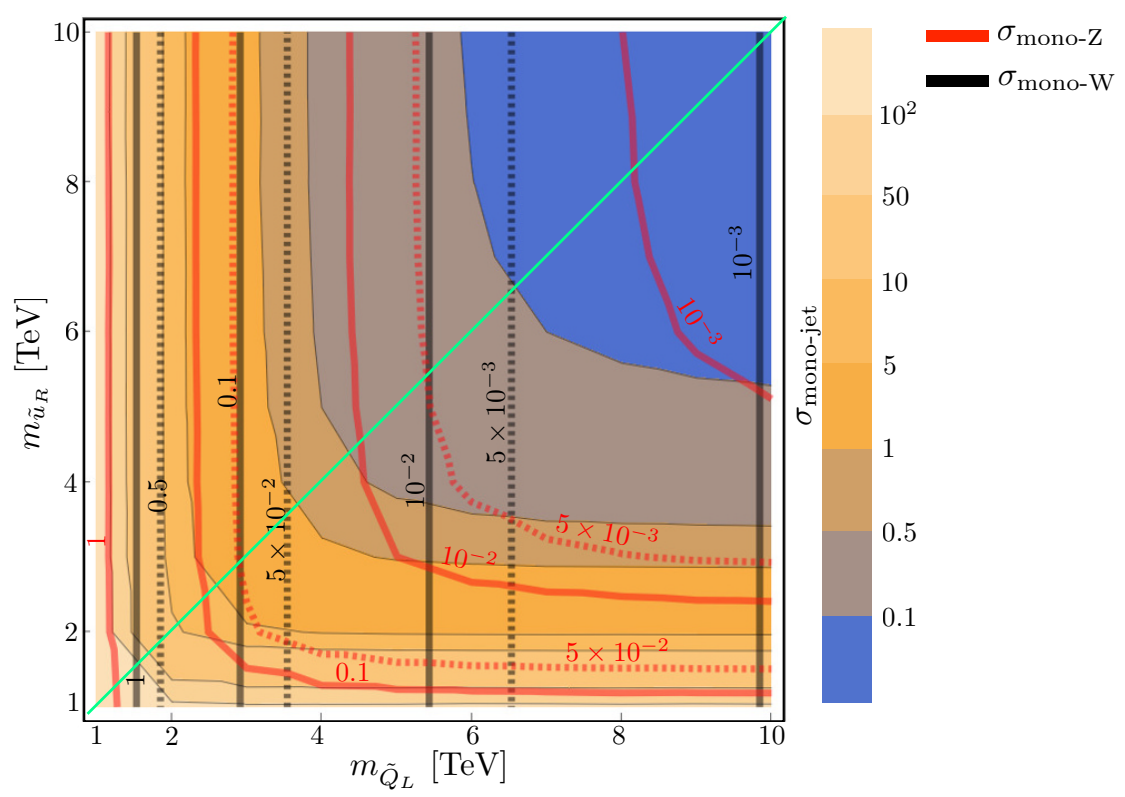

Figure 10. Contour plot of $m_{\widetilde{Q}_{L}}$ vs $m_{\widetilde{u}_{R}}$ displaying mono- $W$, mono- $Z$, and mono-jet cross sections in fb for $\lambda_{Q_{L}}=\lambda_{u_{R}}=1, \lambda_{d_{R}}=\lambda_{4}=0$, and $m_{\chi}=5 \mathrm{GeV}$, and points along the diagonal line represents the simplified model where $\widetilde{u}_{R}$ and $\widetilde{Q}_{L}$ are degenerate with equal coupling constants to quarks.

The dependence on the mediator masses, and the mono- $X$ cross sections in fb are illustrated in the contour plot of figure 10 which shows $m_{\widetilde{Q}_{L}}$ versus $m_{\widetilde{u}_{R}}$ for the case 
when $\lambda_{Q_{L}}=\lambda_{u_{R}}=1, \lambda_{d_{R}}=\lambda_{4}=0$ and $m_{\chi}=5 \mathrm{GeV}$. Note that the simplified model case of universal mediator mass and coupling to $\mathrm{RH}$ and $\mathrm{LH}$ up-quarks is found along the diagonal line starting from the origin, and the case when only one kind of mediator exists is approximated along the lines when either $m_{\widetilde{Q}_{L}}$ or $m_{\widetilde{u}_{R}}$ is $10 \mathrm{TeV}$. Previous studies have shown that failing to account for $\mathrm{SU}(2)_{L}$ gauge invariance has lead to an apparent enhancement of the mono- $W$ signature compared to the mono-jet [15, 63], and while our model is $\mathrm{SU}(2)_{L}$ gauge invariant and avoids this problem, the isospin violation from the $\mathrm{LH}$ and $\mathrm{RH}$ couplings leads to certain choices of the parameter space where the mono-jet to mono- $W$ ratio is enhanced by nearly three orders of magnitude as seen in the contour plot of figure 10. Thus, while no large enhancement of the mono- $W$ signature can be seen, there is a potentially very large effect in the ratio of the mono- $W$ and mono-jet signatures which originates in these generic isospin violating terms and is only visible when the free parameters of the simplified model are loosened from the usual assumptions for the $t$ channel model. Specifically, take a point along the diagonal where $m_{\widetilde{Q}_{L}} \approx m_{\widetilde{u}_{R}}=2 \mathrm{TeV}$ and shift this point along a line where one of the $m_{\widetilde{u}_{R}}=2 \mathrm{TeV}$ and $m_{\widetilde{Q}_{L}}>2 \mathrm{TeV}$, and the mono-jet goes through one order of magnitude change, mono- $W$ goes through two orders of magnitude, and mono- $Z$ varies by a factor of roughly two. Thus the parameter space between the case where mediators are treated near universally, compared to the case where all but the $m_{\widetilde{u}_{R}}$ is too heavy to find at a collider, has a wide range of intermediate predictions that significantly complicate the interpretation of a simplified model constraint derived from LHC data. Despite the increased number of free parameters these large deviations in mono- $X$ signature for different assumptions occurs even when the number of free parameters are restricted such that $\lambda_{4}=0, \widetilde{u}_{R}$ is the lightest $t$-channel scalar, $\lambda_{Q_{L}} \approx 1$, and $\widetilde{d}_{R}$ can be entirely integrated out. Figure 11 shows the case where $\widetilde{d}_{R}$ contribution is added with fixed $\lambda_{d_{R}}=1$ and $m_{\widetilde{d}_{R}}=3 \mathrm{TeV}$. For heavier $\widetilde{u}_{R}$, the mono-jet cross sections is modified, but the mono- $Z$ cross sections are ostensibly the same as the previous case. Note that the point where $m_{\widetilde{u}_{R}}=m_{\widetilde{Q}_{L}}$ in figure 11 is equivalent to the simplified model found in ref. [24] where $m_{\text {mediator }}=3 \mathrm{TeV}$. Again, notice that deviations from this point do lead to significant variation in mono- $X$ cross sections. Importantly, the contour plots of our model allow a practitioner to determine specifically which parameter spaces have overlapping mono- $X$ predictions, to within a factor of 2 , simply by looking at which regions overlap. Figure 12 shows the case where $m_{\chi}=300 \mathrm{GeV}$, while other parameters are the same as those in figure 10. As discussed in the section on direct detection, the $10 \mathrm{GeV}<m_{\chi}<1 \mathrm{TeV}$ mass range is ruled out for $m_{\text {mediator }} \leq 10 \mathrm{TeV}$. However, as described previously, these constraints can be lifted if we change our assumptions about the nature of $\chi$. That is, if $\chi$ is a small component of the cosmological DM, or if it is merely stable for long enough to escape detection at a collider the mass of $\chi$ can be changed. For example, we find that if $m_{\chi}=300 \mathrm{GeV}$ and if we assume that there is another species of DM that makes up the majority of the cosmological DM, this mass region is no longer excluded by direct detection. This different DM mass range has notable effects on the mono- $X$ cross sections as shown in figure 12 .

Normalized event distributions of $p_{T}$ for the mono-jet and mono- $W$ signatures are plotted in figure 13 and figure 14 . For the $p_{T}$ distribution of the mono- $W$ events, the 


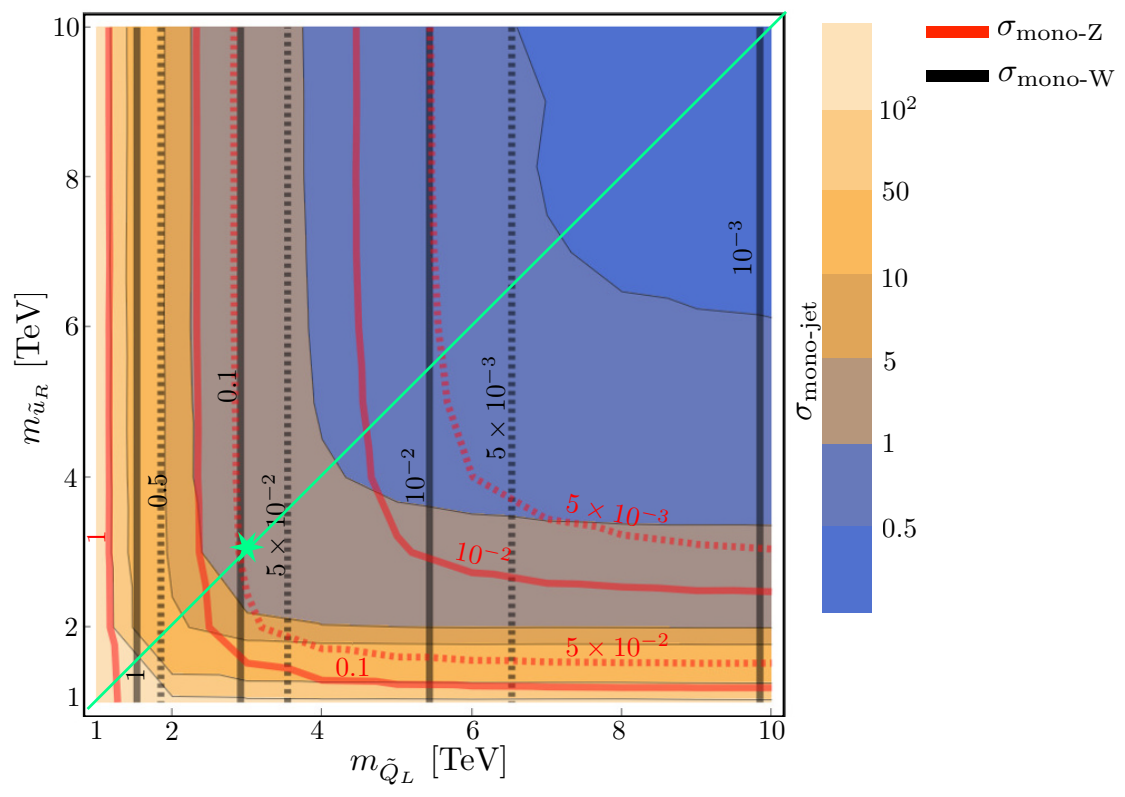

Figure 11. The same as figure 10, but with $\lambda_{d_{R}}=1$ and $m_{\widetilde{d}_{R}}=3 \mathrm{TeV}$, where points along the diagonal line represents the simplified model where $\widetilde{u}_{R}$ and $\widetilde{Q}_{L}$ are degenerate with equal coupling constants to quarks and the star is the simplified model in ref. [24] with $m_{\text {med }}=3 \mathrm{TeV}$ and $m_{\chi}=5 \mathrm{GeV}$.

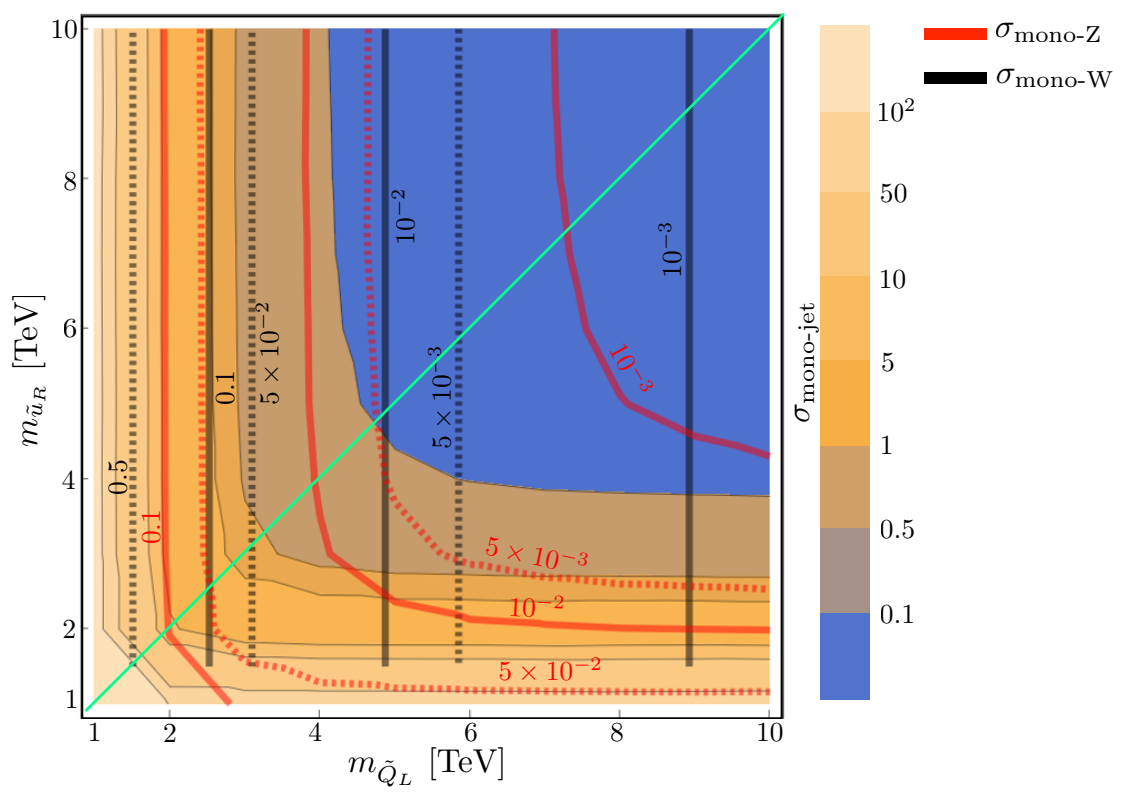

Figure 12. The same as figure 10 , but with $m_{\chi}=300 \mathrm{GeV}$. 




Figure 13. Normalized distributions for mono- $W p_{T}$ when $\lambda_{Q_{L}}=1, \lambda_{4}=0$, and $m_{\chi}=5 \mathrm{GeV}$ at parton-level.

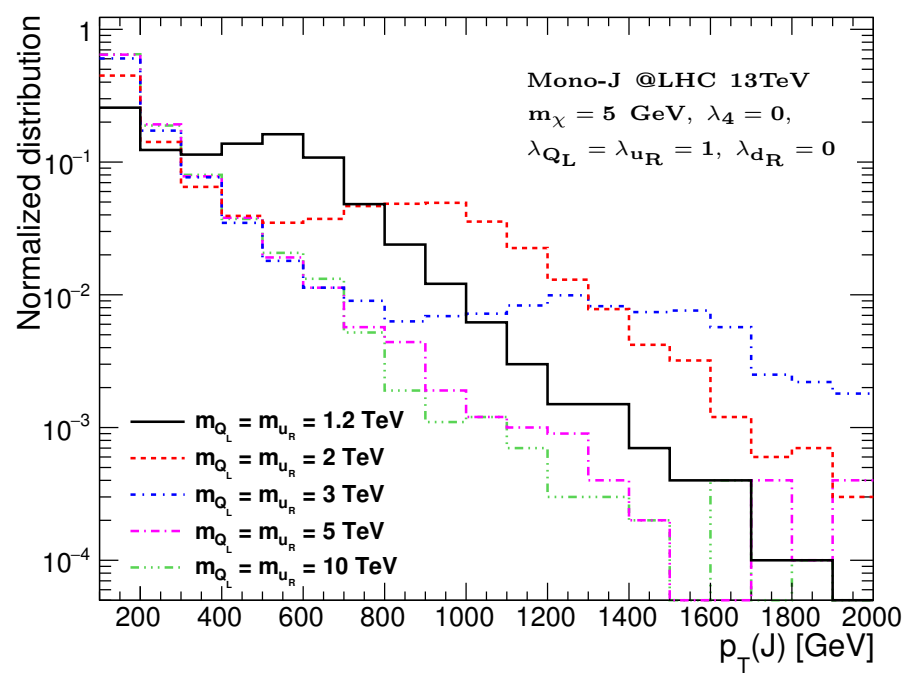

Figure 14. Normalized distributions of mono-jet $p_{T}$ when $\lambda_{Q_{L}}=\lambda_{u_{R}}=1, \lambda_{d_{R}}=\lambda_{4}=0$, and $m_{\chi}=5 \mathrm{GeV}$ at parton-level.

UV theory has a broader tail in the very high $p_{T}(>500 \mathrm{GeV})$ region, because the small squark virtuality configurations become relatively important. On the other hand, the monojet events have significant peaks centered at around $m_{\widetilde{Q}_{L}} / 2$ due to the Jacobian peak in the scalar decays into a quark and DM. In figure 15, we show distributions of letponic observables in mono- $W$ events after Delphes detector simulation. The leptonic observables that are plotted are, moving left-to-right in figure 15: lepton $p_{T}$, missing $E_{T}$ (MET), and the transverse mass $M_{T}^{\ell}=\sqrt{2 p_{T}^{\ell} E_{T}(1-\cos \Delta \phi)}$ where $\Delta \phi$ is an opening angle of the 


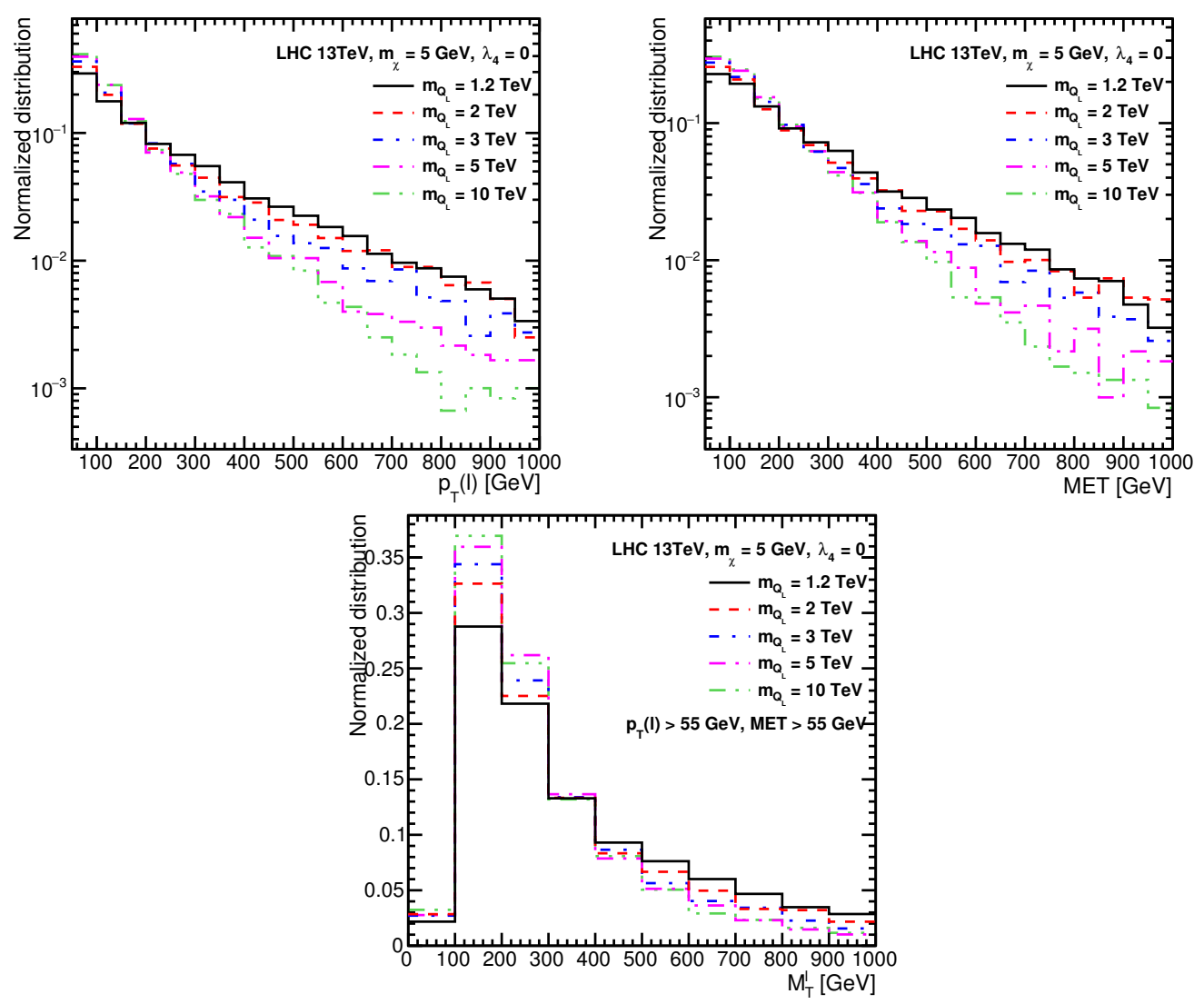

Figure 15. Leptonic observables in mono- $W$ with Delphes simulation for $\lambda_{4}=0$ and $m_{\chi}=5 \mathrm{GeV}$.

lepton and missing momentum in the transverse plane. We find the $p_{T}$ distribution of the charged lepton and $\mathbb{E}_{T}$ distribution are similar with the $p_{T}$ distribution of $W$. In addition, $M_{T}^{\ell}$ distributions are also different for different scalar mediator masses. Thus, these leptonic observables can be used to determine the internal parameters of the UV-complete theory, if enough events are collected at the LHC Run-II.

\section{Summary}

The typical approach to investigating DM at colliders, particularly the colored $t$-channel scalars, is to make basic assumptions about the particle content and the coupling. Namely, doublet-only, up-like signlet only, down-like singlet only, or universal coupling and mass for all $t$-channel scalars. Direct detection strongly constrains the mass of dark matter such that any mass within the range of $10 \mathrm{GeV} \leq m_{\chi} \leq 1000 \mathrm{GeV}$ for a wide range of colored scalars is ruled out, and there is a tension between the relic density and direct detection for simplified models that do not include information about the coupling to leptons. Additionally, both the relic denisty and direct detection constraints can be changed if there are other species of DM, or if the $\chi$ particle is stable enough to escape a detector if it is produced at a collider, and thus a $m_{\chi}$ within the range where it is ruled out by direct 
detection may be observed at the LHC in such a scenario, particularly we have looked at the case when $m_{\chi}=300 \mathrm{GeV}$.

For many of the parameter choices presented in this study the usual assumptions used for simplified models are justified, however for particular choices of parameters there are striking variations in the experimental predictions. In general, running effects make it inappropriate to restrict the model to a single direct detection operator defined at high energy [23]. Generically, even if the UV-complete model produced a single set of operators, such as D5 or D7 as defined in ref. [73], these running effects would mix the operators making the direct comparison of LHC Run-I searches to direct detection inappropriate even under the assumption that DM is a single species and $\chi$ is absolutely stable. Moreover, a UV-complete model that respects the full SM gauge symmetry, and properly accounts for $\mathrm{SU}(2)_{L}$ invariance could have multiple relevant operators, as discussed in this paper. These running effects generically generate isospin violating effects in direct detection, but additional effects can be seen in detectors when $\lambda_{u_{R}} \neq \lambda_{d_{R}}$ as is generally the case in a UVcomplete model and, in fact, $\lambda_{u_{R}} \neq \lambda_{d_{R}}$ is required in our model to counter-act the generic isospin violating effects seen in ref. [23], thus a seeming null result in isospin violation at a direct detection experiment may actually imply such isospin violation exists at higher energies. Of particular note is the large effect these different assumptions can have on collider signatures, where for a given $m_{\widetilde{Q}_{L}}$ and $\lambda_{Q_{L}}$ the mono- $W$ signature is the same, but the mono-jet signature can vary by as much as three orders of magnitude. In fact, as parameters are varied from a previously considered simplified model to a parameter space that approximates another simplified model, these mono- $X$ signatures generically change by significant amounts. From a practitioner's perspective, using a simplified model with a design philosophy similar to ours yields contour plots of the various mono- $X$ cross sections which allow the efficient determination of where in the parameter space simplified models have overlapping or distinct predictions, and thus where an LHC signature could, or could not, uniquely determine the properties of the dark sector. Thus, while simplified models are still an important tool to understand DM physics at colliders, the broader framework of using the full SM gauge symmetry as discussed above, and allowing for more parameters to vary independently, allows the investigation of parameter space of mono- $X$ signatures that are otherwise ignored.

\section{Acknowledgments}

We are grateful to Oliver Buchmüller, Patrick Fox, K. Hahn and Lian-Tao Wang, for useful discussions on the simplified DM models. This work is supported in part by National Research Foundation of Korea (NRF) Research Grant NRF-2015R1A2A1A05001869, and by the NRF grant funded by the Korea government (MSIP) (No. 2009-0083526) through Korea Neutrino Research Center at Seoul National University (PK), and also by IBS under the project code, IBS-R018-D1 (MP).

Open Access. This article is distributed under the terms of the Creative Commons Attribution License (CC-BY 4.0), which permits any use, distribution and reproduction in any medium, provided the original author(s) and source are credited. 


\section{References}

[1] G. Bertone, D. Hooper and J. Silk, Particle dark matter: Evidence, candidates and constraints, Phys. Rept. 405 (2005) 279 [hep-ph/0404175] [INSPIRE].

[2] ATLAS collaboration, Search for new phenomena in final states with an energetic jet and large missing transverse momentum in pp collisions at $\sqrt{s}=8$ TeV with the ATLAS detector, Eur. Phys. J. C 75 (2015) 299 [arXiv:1502.01518] [InSPIRE].

[3] ATLAS collaboration, Search for new particles in events with one lepton and missing transverse momentum in pp collisions at $\sqrt{s}=8$ TeV with the ATLAS detector, JHEP 09 (2014) 037 [arXiv: 1407.7494] [INSPIRE].

[4] ATLAS collaboration, Search for dark matter in events with a $Z$ boson and missing transverse momentum in pp collisions at $\sqrt{s}=8$ TeV with the ATLAS detector, Phys. Rev. D 90 (2014) 012004 [arXiv:1404.0051] [INSPIRE].

[5] CMS collaboration, Search for physics beyond the standard model in final states with a lepton and missing transverse energy in proton-proton collisions at $\sqrt{s}=8$ TeV, Phys. Rev. D 91 (2015) 092005 [arXiv: 1408.2745] [INSPIRE].

[6] ATLAS collaboration, Search for dark matter in events with a hadronically decaying $W$ or $Z$ boson and missing transverse momentum in pp collisions at $\sqrt{s}=8$ TeV with the ATLAS detector, Phys. Rev. Lett. 112 (2014) 041802 [arXiv: 1309.4017] [InSPIRE].

[7] A. Askew, S. Chauhan, B. Penning, W. Shepherd and M. Tripathi, Searching for Dark Matter at Hadron Colliders, Int. J. Mod. Phys. A 29 (2014) 1430041 [arXiv:1406.5662] [InSPIRE].

[8] O. Buchmueller, M.J. Dolan and C. McCabe, Beyond Effective Field Theory for Dark Matter Searches at the LHC, JHEP 01 (2014) 025 [arXiv: 1308.6799] [INSPIRE].

[9] G. Busoni, A. De Simone, T. Jacques, E. Morgante and A. Riotto, On the Validity of the Effective Field Theory for Dark Matter Searches at the LHC Part III: Analysis for the t-channel, JCAP 09 (2014) 022 [arXiv: 1405.3101] [INSPIRE].

[10] J. Abdallah et al., Simplified Models for Dark Matter and Missing Energy Searches at the $L H C$, arXiv:1409.2893 [INSPIRE].

[11] LHC New Physics Working Group collaboration, D. Alves, Simplified Models for LHC New Physics Searches, J. Phys. G 39 (2012) 105005 [arXiv:1105.2838] [InSPIRE].

[12] J. Abdallah et al., Simplified Models for Dark Matter Searches at the LHC, Phys. Dark Univ. 9-10 (2015) 8 [arXiv:1506.03116] [INSPIRE].

[13] F. Kahlhoefer, K. Schmidt-Hoberg, T. Schwetz and S. Vogl, Implications of unitarity and gauge invariance for simplified dark matter models, JHEP 02 (2016) 016 [arXiv: 1510.02110] [INSPIRE].

[14] S. Baek, P. Ko, M. Park, W.-I. Park and C. Yu, Beyond the Dark matter effective field theory and a simplified model approach at colliders, Phys. Lett. B 756 (2016) 289 [arXiv: 1506.06556] [INSPIRE].

[15] N.F. Bell, Y. Cai, J.B. Dent, R.K. Leane and T.J. Weiler, Dark matter at the LHC: Effective field theories and gauge invariance, Phys. Rev. D 92 (2015) 053008 [arXiv:1503.07874] [INSPIRE].

[16] S. Baek, P. Ko and W.-I. Park, Search for the Higgs portal to a singlet fermionic dark matter at the LHC, JHEP 02 (2012) 047 [arXiv: 1112.1847] [INSPIRE]. 
[17] P. Ko and J. Li, Interference effects of two scalar boson propagators on the LHC search for the singlet fermion DM, Phys. Lett. B 765 (2017) 53 [arXiv:1610.03997] [InSPIRE].

[18] P. Ko and H. Yokoya, Search for Higgs portal DM at the ILC, JHEP 08 (2016) 109 [arXiv: 1603.04737] [INSPIRE].

[19] A.J. Buras, Flavour Theory: 2009, PoS(EPS-HEP 2009) 024 [arXiv:0910.1032] [INSPIRE].

[20] S. Jung, P. Ko, Y.W. Yoon and C. Yu, Renormalization group-induced phenomena of top pairs from four-quark effective operators, JHEP 08 (2014) 120 [arXiv:1406.4570] [INSPIRE].

[21] G. Busoni et al., Recommendations on presenting LHC searches for missing transverse energy signals using simplified s-channel models of dark matter, arXiv:1603.04156 [INSPIRE].

[22] F. D'Eramo, B.J. Kavanagh and P. Panci, You can hide but you have to run: direct detection with vector mediators, JHEP 08 (2016) 111 [arXiv:1605.04917] [INSPIRE].

[23] F. D'Eramo and M. Procura, Connecting Dark Matter UV Complete Models to Direct Detection Rates via Effective Field Theory, JHEP 04 (2015) 054 [arXiv:1411.3342] [INSPIRE].

[24] D. Abercrombie et al., Dark Matter Benchmark Models for Early LHC Run-2 Searches: Report of the ATLAS/CMS Dark Matter Forum, arXiv:1507.00966 [INSPIRE].

[25] Y. Bai and T.M.P. Tait, Searches with Mono-Leptons, Phys. Lett. B 723 (2013) 384 [arXiv: 1208.4361] [INSPIRE].

[26] S. Baek, P. Ko and W.-I. Park, Singlet Portal Extensions of the Standard Seesaw Models to a Dark Sector with Local Dark Symmetry, JHEP 07 (2013) 013 [arXiv: 1303.4280] [INSPIRE].

[27] S. Baek, P. Ko and W.-I. Park, Hidden sector monopole, vector dark matter and dark radiation with Higgs portal, JCAP 10 (2014) 067 [arXiv:1311.1035] [INSPIRE].

[28] P. Ko and Y. Tang, Self-interacting scalar dark matter with local $Z_{3}$ symmetry, JCAP 05 (2014) 047 [arXiv: 1402.6449] [INSPIRE].

[29] P. Ko and Y. Tang, $\nu \Lambda M D M:$ A model for sterile neutrino and dark matter reconciles cosmological and neutrino oscillation data after BICEP2, Phys. Lett. B 739 (2014) 62 [arXiv: 1404.0236] [INSPIRE].

[30] P. Ko and W.-I. Park, Higgs-portal assisted Higgs inflation with a sizeable tensor-to-scalar ratio, arXiv:1405.1635 [INSPIRE].

[31] P. Ko and Y. Tang, Galactic center $\gamma$-ray excess in hidden sector DM models with dark gauge symmetries: local $Z_{3}$ symmetry as an example, JCAP 01 (2015) 023 [arXiv:1407.5492] [INSPIRE].

[32] S. Baek, P. Ko and W.-I. Park, Local $Z_{2}$ scalar dark matter model confronting galactic GeV-scale $\gamma$-ray, Phys. Lett. B 747 (2015) 255 [arXiv: 1407.6588] [INSPIRE].

[33] P. Ko and Y. Tang, AMSO2 positron excess from decaying fermion DM with local dark gauge symmetry, Phys. Lett. B 741 (2015) 284 [arXiv:1410.7657] [INSPIRE].

[34] P. Ko and Y. Tang, Dark Higgs Channel for FERMI GeV $\gamma$-ray Excess, JCAP 02 (2016) 011 [arXiv: 1504.03908] [INSPIRE].

[35] P. Ko and Y. Tang, IceCube Events from Heavy DM decays through the Right-handed Neutrino Portal, Phys. Lett. B 751 (2015) 81 [arXiv: 1508.02500] [InSPIRE]. 
[36] A. Alves, A. Berlin, S. Profumo and F.S. Queiroz, Dirac-fermionic dark matter in U(1) $X$ models, JHEP 10 (2015) 076 [arXiv:1506.06767] [INSPIRE].

[37] A. Alves, A. Berlin, S. Profumo and F.S. Queiroz, Dark Matter Complementarity and the Z' Portal, Phys. Rev. D 92 (2015) 083004 [arXiv:1501.03490] [inSPIRE].

[38] A. Alves, S. Profumo and F.S. Queiroz, The dark $Z^{\prime}$ portal: direct, indirect and collider searches, JHEP 04 (2014) 063 [arXiv: 1312.5281] [INSPIRE].

[39] S. Baek, P. Ko, W.-I. Park and E. Senaha, Vacuum structure and stability of a singlet fermion dark matter model with a singlet scalar messenger, JHEP 11 (2012) 116 [arXiv: 1209.4163] [INSPIRE].

[40] S. Baek, P. Ko, W.-I. Park and E. Senaha, Higgs Portal Vector Dark Matter : Revisited, JHEP 05 (2013) 036 [arXiv:1212.2131] [INSPIRE].

[41] P. Ko, W.-I. Park and Y. Tang, Higgs portal vector dark matter for $\mathrm{GeV}$ scale $\gamma$-ray excess from galactic center, JCAP 09 (2014) 013 [arXiv: 1404.5257] [INSPIRE].

[42] S. Baek, P. Ko and W.-I. Park, Invisible Higgs Decay Width vs. Dark Matter Direct Detection Cross Section in Higgs Portal Dark Matter Models, Phys. Rev. D 90 (2014) 055014 [arXiv: 1405.3530] [INSPIRE].

[43] H. An, L.-T. Wang and H. Zhang, Dark matter with t-channel mediator: a simple step beyond contact interaction, Phys. Rev. D 89 (2014) 115014 [arXiv:1308.0592] [INSPIRE].

[44] M. Papucci, A. Vichi and K.M. Zurek, Monojet versus the rest of the world I: t-channel models, JHEP 11 (2014) 024 [arXiv:1402.2285] [INSPIRE].

[45] A. DiFranzo, K.I. Nagao, A. Rajaraman and T.M.P. Tait, Simplified Models for Dark Matter Interacting with Quarks, JHEP 11 (2013) 014 [Erratum ibid. 1401 (2014) 162] [arXiv:1308.2679] [INSPIRE].

[46] S. Chang, R. Edezhath, J. Hutchinson and M. Luty, Effective WIMPs, Phys. Rev. D 89 (2014) 015011 [arXiv:1307.8120] [INSPIRE].

[47] N.F. Bell, J.B. Dent, A.J. Galea, T.D. Jacques, L.M. Krauss and T.J. Weiler, Searching for Dark Matter at the LHC with a Mono-Z, Phys. Rev. D 86 (2012) 096011 [arXiv:1209.0231] [INSPIRE].

[48] A.J. Brennan, M.F. McDonald, J. Gramling and T.D. Jacques, Collide and Conquer: Constraints on Simplified Dark Matter Models using Mono-X Collider Searches, JHEP 05 (2016) 112 [arXiv: 1603.01366] [INSPIRE].

[49] A. Choudhury, K. Kowalska, L. Roszkowski, E.M. Sessolo and A.J. Williams, Less-simplified models of dark matter for direct detection and the LHC, JHEP 04 (2016) 182 [arXiv: 1509.05771] [INSPIRE].

[50] D. Racco, A. Wulzer and F. Zwirner, Robust collider limits on heavy-mediator Dark Matter, JHEP 05 (2015) 009 [arXiv: 1502.04701] [INSPIRE].

[51] M. Drees and M. Nojiri, Neutralino-nucleon scattering revisited, Phys. Rev. D 48 (1993) 3483 [hep-ph/9307208] [INSPIRE].

[52] J. Hisano, K. Ishiwata and N. Nagata, Gluon contribution to the dark matter direct detection, Phys. Rev. D 82 (2010) 115007 [arXiv:1007.2601] [InSPIRE].

[53] P. Gondolo and S. Scopel, On the sbottom resonance in dark matter scattering, JCAP 10 (2013) 032 [arXiv:1307.4481] [INSPIRE]. 
[54] A. Ibarra and S. Wild, Dirac dark matter with a charged mediator: a comprehensive one-loop analysis of the direct detection phenomenology, JCAP 05 (2015) 047 [arXiv: 1503.03382] [INSPIRE].

[55] A. Crivellin, F. D'Eramo and M. Procura, New Constraints on Dark Matter Effective Theories from Standard Model Loops, Phys. Rev. Lett. 112 (2014) 191304 [arXiv: 1402.1173] [INSPIRE].

[56] J. Hisano, K. Ishiwata, N. Nagata and T. Takesako, Direct Detection of Electroweak-Interacting Dark Matter, JHEP 07 (2011) 005 [arXiv:1104.0228] [INSPIRE].

[57] J. Hisano, R. Nagai and N. Nagata, Effective Theories for Dark Matter Nucleon Scattering, JHEP 05 (2015) 037 [arXiv: 1502.02244] [INSPIRE].

[58] LUX collaboration, D.S. Akerib et al., First results from the LUX dark matter experiment at the Sanford Underground Research Facility, Phys. Rev. Lett. 112 (2014) 091303 [arXiv: 1310.8214] [INSPIRE].

[59] LUX collaboration, D.S. Akerib et al., Improved Limits on Scattering of Weakly Interacting Massive Particles from Reanalysis of 2013 LUX Data, Phys. Rev. Lett. 116 (2016) 161301 [arXiv: 1512.03506] [INSPIRE].

[60] Q.-H. Cao, E. Ma, J. Wudka and C.P. Yuan, Multipartite dark matter, arXiv:0711.3881 [INSPIRE].

[61] Fermi-LAT collaboration, M. Ajello et al., Search for Spectral Irregularities due to Photon-Axionlike-Particle Oscillations with the Fermi Large Area Telescope, Phys. Rev. Lett. 116 (2016) 161101 [arXiv:1603.06978] [INSPIRE].

[62] SuperCDMS collaboration, R. Agnese et al., Search for Low-Mass Weakly Interacting Massive Particles Using Voltage-Assisted Calorimetric Ionization Detection in the SuperCDMS Experiment, Phys. Rev. Lett. 112 (2014) 041302 [arXiv:1309.3259] [INSPIRE].

[63] N.F. Bell, Y. Cai and R.K. Leane, Mono-W Dark Matter Signals at the LHC: Simplified Model Analysis, JCAP 01 (2016) 051 [arXiv:1512.00476] [INSPIRE].

[64] N.F. Bell, J.B. Dent, T.D. Jacques and T.J. Weiler, W/Z Bremsstrahlung as the Dominant Annihilation Channel for Dark Matter, Phys. Rev. D 83 (2011) 013001 [arXiv:1009. 2584] [INSPIRE].

[65] C.C. Nishi, Simple derivation of general Fierz-like identities, Am. J. Phys. 73 (2005) 1160 [hep-ph/0412245] [INSPIRE].

[66] A. Alloul, N.D. Christensen, C. Degrande, C. Duhr and B. Fuks, FeynRules 2.0 - A complete toolbox for tree-level phenomenology, Comput. Phys. Commun. 185 (2014) 2250 [arXiv:1310.1921] [INSPIRE].

[67] J. Alwall et al., The automated computation of tree-level and next-to-leading order differential cross sections and their matching to parton shower simulations, JHEP 07 (2014) 079 [arXiv: 1405.0301] [INSPIRE].

[68] R.D. Ball et al., Parton distributions with LHC data, Nucl. Phys. B 867 (2013) 244 [arXiv:1207.1303] [INSPIRE].

[69] T. Sjöstrand, S. Mrenna and P.Z. Skands, PYTHIA 6.4 Physics and Manual, JHEP 05 (2006) 026 [hep-ph/0603175] [INSPIRE]. 
[70] DELPHES 3 collaboration, J. de Favereau et al., DELPHES 3, A modular framework for fast simulation of a generic collider experiment, JHEP 02 (2014) 057 [arXiv:1307.6346] [INSPIRE].

[71] R. Brun and F. Rademakers, Root - an object oriented data analysis framework, Nucl. Instrum. Methods Phys. Res. A 389 (1997) 81.

[72] ATLAS collaboration, Search for squarks and gluinos in final states with jets and missing transverse momentum at $\sqrt{s}=13$ TeV with the ATLAS detector, ATLAS-CONF-2015-062.

[73] J. Goodman, M. Ibe, A. Rajaraman, W. Shepherd, T.M.P. Tait and H.-B. Yu, Constraints on Dark Matter from Colliders, Phys. Rev. D 82 (2010) 116010 [arXiv:1008.1783] [InSPIRE]. 